\title{
Direct entrainment and detrainment rate distributions of individual shallow cumulus clouds in an LES
}

\author{
J. T. Dawe and P. H. Austin \\ Department of Earth, Ocean, and Atmospheric Sciences, University of British Columbia, Room 2020, Earth Sciences \\ Building, 2207 Main Mall, Vancouver, BC, V6T 1Z4, Canada
}

Correspondence to: J. T. Dawe (jdawe@eos.ubc.ca)

Received: 23 January 2013 - Published in Atmos. Chem. Phys. Discuss.: 27 February 2013

Revised: 1 June 2013 - Accepted: 21 June 2013 - Published: 13 August 2013

\begin{abstract}
Probability distribution functions of shallow cumulus cloud core entrainment and detrainment rates are calculated using 4362 individual cumulus clouds isolated from LES (large eddy simulation) using a cloud tracking algorithm. Calculation of the mutual information between fractional entrainment/detrainment and a variety of mean cloud core properties suggests that fractional entrainment rate is best predicted by the mean cloud buoyancy $B$ and the environmental buoyancy lapse rate $\mathrm{d} \overline{\theta_{\rho}} / \mathrm{d} z$ at that level, while fractional detrainment is best predicted by the mean vertical velocity $w$ and the critical mixing fraction $\chi_{\mathrm{c}}$. Fractional entrainment and detrainment rates are relatively insensitive to cloud core horizontal area, and the perimeter of horizontal cloud core sections display an $a^{0.73}$ dependence. This implies that cloud core mass entrainment flux $E$ is proportional to cloud core cross-sectional area instead of cloud core surface area, as is generally assumed. Empirical best-fit relations for $\epsilon\left(B, \mathrm{~d} \overline{\theta_{\rho}} / \mathrm{d} z\right)$ and $\delta\left(w, \chi_{\mathrm{c}}\right)$ are found for both individual shallow cumulus clouds and cloud ensembles. It is found that clouds with high buoyancy in strong stratification experience low entrainment rates, while clouds with high vertical velocities and critical mixing fractions experience low detrainment rates.
\end{abstract}

\section{Introduction}

Shallow cumulus clouds, sometimes referred to as tradewind cumulus, occur in the tropics as a transitional state between stratus decks, which occur in strongly stratified downwelling regions, and deep cumulus clouds, which occur in weakly stratified upwelling regions. Shallow cumulus reach heights of $2-3 \mathrm{~km}$, transporting heat and moisture upward which erodes the inversion stratification and preconditions the atmosphere for deep convection. Biases in the parameterization of shallow cumulus in general circulation models (GCMs) have impacts on the distribution and intensity of deep convection, which can result in poor representations of the Hadley and Walker circulations (Stevens, 2005).

Additionally, shallow cumulus serve as a test for GCM cloud parameterizations, which in general have been developed for stratus or deep cumulus. Because of this, several shallow cumulus test cases, based upon field campaigns, have been created by the Global Energy and Water Cycle Experiment (GEWEX) Cloud System Studies (GCSS; Randall et al., 2003) boundary layer cloud group, suitable for modelling via large eddy simulation (LES; Stevens et al., 2001; Brown et al., 2002; Siebesma et al., 2003; vanZanten et al., 2011). Much of this work has focused on the entrainment and detrainment rates of shallow cumulus, which strongly affect shallow cumulus properties and constitute one of the largest sources of uncertainty in GCMs (Sanderson et al., 2008; Klocke et al., 2011).

Entrainment and detrainment of mass is defined as the rate at which mass crosses into (entrainment) or out of (detrainment) some region in a fluid, such as the region containing condensed liquid water (i.e. a cloud). The entrainment and detrainment rates of a cloud at a given height can be formally defined as (Siebesma, 1998) 


$$
\begin{aligned}
& E=-\oint_{\hat{\boldsymbol{n}} \cdot\left(\boldsymbol{u}-\boldsymbol{u}_{i}\right)<0} \rho \hat{\boldsymbol{n}} \cdot\left(\boldsymbol{u}-\boldsymbol{u}_{i}\right) \mathrm{d} l, \\
& D=\oint_{\hat{\boldsymbol{n}} \cdot\left(\boldsymbol{u}-\boldsymbol{u}_{i}\right)>0} \rho \hat{\boldsymbol{n}} \cdot\left(\boldsymbol{u}-\boldsymbol{u}_{i}\right) \mathrm{d} l,
\end{aligned}
$$

where $E$ and $D$ are the entrainment and detrainment rates $\left(\mathrm{kg} \mathrm{m}^{-1} \mathrm{~s}^{-1}\right), \rho$ is the density of air $\left(\mathrm{kg} \mathrm{m}^{-3}\right), \boldsymbol{u}$ is the velocity of the air $\left(\mathrm{m} \mathrm{s}^{-1}\right), \boldsymbol{u}_{i}$ is the velocity of the cloud surface $\left(\mathrm{m} \mathrm{s}^{-1}\right), \hat{\boldsymbol{n}}$ is a unit vector directed out of the cloud surface, and the path integral is taken around the cloud surface at a constant vertical level. However, mass entrainment and detrainment are more often represented with the fractional mass entrainment and detrainment rates $\epsilon=E / M$ and $\delta=E / M$ (both $\mathrm{m}^{-1}$ ), where $M=\rho w a$ is the vertical mass flux $\left(\mathrm{kg} \mathrm{s}^{-1}\right), w$ is the vertical velocity $\left(\mathrm{m} \mathrm{s}^{-1}\right)$, and $a$ is the cross-sectional area $\left(\mathrm{m}^{2}\right)$ of the cloud. These can be thought of as the fraction of the cloud mass that is being entrained and detrained per metre of rise through the cloud.

Many parameterizations of cumulus entrainment and detrainment rates have been proposed and tested against LES output (de Rooy et al., 2012). Turner (1963) proposed a simple scaling for entrainment as being proportional to the cloud vertical velocity times the perimeter of a cross section. This results in the fractional entrainment at a given height being inversely proportional to the cloud radius (assuming the cloud cross section is roughly circular). This has served as the basis of many parameterizations (Arakawa and Schubert, 1974; Tiedtke, 1989; Kain and Fritsch, 1990; Wagner and Graf, 2010), some of which make the further assumption that variations in the effective radius of the cloud field are negligible and so $\epsilon$ and $\delta$ can be treated as constants (Tiedtke, 1989; Bretherton and Park, 2008). Others parameterize the effective cloud radius as proportional to the height of cloud top (Bretherton et al., 2004), or simply allow $\epsilon$ to be inversely proportional to height (de Rooy and Siebesma, 2008).

Buoyancy sorting schemes allow entrainment and detrainment to depend on the properties of cloud and environment by assuming that cloud parcels experience a range of mixing rates, and the parcels which become negatively buoyant as a result of this mixing detrain from the cloud plume (Kain and Fritsch, 1990; Bretherton et al., 2004; de Rooy and Siebesma, 2008). The critical mixing fraction $\chi_{c}-$ the fraction of environmental air in a mixture of cloudy and environmental air needed to make the mixture neutrally buoyant - is the primary control on entrainment and detrainment in these parameterizations, with larger $\chi_{\mathrm{c}}$ resulting in larger $\epsilon$ and smaller $\delta$. In a similar spirit, Bechtold et al. (2008) and Stirling and Stratton (2012) allow entrainment to depend directly upon the atmospheric specific humidity.

Several parameterizations use various arguments to link entrainment and detrainment to the dynamic variables of the clouds. Neggers et al. (2002) proposed an inverse relation- ship between $\epsilon$ and vertical velocity $w$. Using arguments concerning the rate turbulent kinetic energy is produced in the cloud, Gregory (2001) proposed $\epsilon \propto B / w^{2}$, where $B$ is the buoyancy of the cloud $\left(\mathrm{m} \mathrm{s}^{-2}\right)$. von Salzen and McFarlane (2002) use $\epsilon \propto \mathrm{d} B / \mathrm{d} z$, while de Rooy and Siebesma (2010) present relations for $\epsilon$ and $\delta$ dependent on $B / w^{2}, w^{-1} \mathrm{~d} w / \mathrm{d} z$, and $a^{-1} \mathrm{~d} a / \mathrm{d} z$. Finally, Romps and Kuang (2010) proposed that entrainment is essentially random, and that entrainment rate should be parameterized as a stochastic process with a set probability of a discrete mixing event occurring for every $L$ metres a parcel rises.

The wide range of parameterization forms present in the literature for the entrainment and detrainment rates suggests the modelling community has not yet reached agreement on which variables are the best predictors of these processes. It is therefore important to develop better ways to test these hypotheses over a wide range of cloud and environmental conditions.

Traditionally entrainment and detrainment rates are diagnosed in LESs using mean cloud field tracer budgets (Siebesma and Cuijpers, 1995). Recently, Romps (2010) and Dawe and Austin (2011a) have developed methods to calculate these rates directly from model velocity, humidity, and temperature fields. These directly calculated entrainment/detrainment rates are $\approx 3$ times larger than those calculated via tracer budgets due to the presence of a shell of recirculated air surrounding the clouds which biases the tracer budget calculations (Dawe and Austin, 2011b). Unlike tracer budget calculations, these new direct calculation methods allow us to easily localize entrainment and detrainment to individual clouds, and provide us with a new way to study the dependence of entrainment and detrainment rates on cloud properties.

Since $\epsilon$ and $\delta$ of the cloud ensemble are the result of the entrainment and detrainment of the individual clouds in the ensemble, studying the entrainment and detrainment of the individual clouds should give some insight into the behaviour of the ensemble. Since a single LES simulates hundreds or thousands of clouds, analysis of individual clouds will produce several orders of magnitude more statistical samples of $\epsilon, \delta$, and other cloud properties from an LES than simply analysing the mean cloud field properties. To this end, this study uses the direct entrainment/detrainment rate calculation method detailed in Dawe and Austin (2011a) and the cloud tracking algorithm detailed in Dawe and Austin (2012) to estimate joint probability distribution functions of fractional entrainment and detrainment rates with a variety of cloud properties for individual LES shallow cumulus clouds. Using measures of the mutual information shared between cloud properties and the fractional entrainment and detrainment rates, we develop a parameterization to predict the mean fractional entrainment and detrainment rates of individual shallow cumulus clouds, and extend this to the prediction of the bulk entrainment and detrainment rates of the cloud ensemble. 


\section{Model description and output data sets}

All LES calculations in this paper were made using the System for Atmospheric Modelling (SAM version 6.8.2; Khairoutdinov and Randall, 2003). Two model runs were performed, configured as standard GCSS cases: a Barbados Oceanographic and Meteorological Experiment (BOMEX; Siebesma et al., 2003) run, and an Atmospheric Radiation Measurement study (ARM; Brown et al., 2002) run. The BOMEX run was performed on a $6.4 \mathrm{~km} \times 6.4 \mathrm{~km}$ horizontal $\times 3.2 \mathrm{~km}$ vertical domain for $6 \mathrm{~h}$, and the first $3 \mathrm{~h}$ of simulation were discarded. The ARM run was performed on a $6.4 \mathrm{~km} \times 6.4 \mathrm{~km} \times 4.5 \mathrm{~km}$ domain and $8.5 \mathrm{~h}$ of output between hour 4.5 and 13 were saved. Both models were run with a $25 \mathrm{~m}$ grid size in all directions and a time step of $1 \mathrm{~s}$. Precipitation was disabled in both runs.

Instantaneous model fields were output each minute, generating 180 snapshots for the BOMEX run and 510 snapshots for the ARM run. Individual cloud histories were then identified from the model outputs using the cloud tracking algorithm detailed in Dawe and Austin (2012). The algorithm identified 2838 individual clouds in the BOMEX run and 1524 clouds in the ARM run.

Note that few of the calculations performed in this paper rely on the time histories of individual clouds, and could have been performed equally well by identifying connected cloudy regions in the model snapshots. Using the cloud tracking algorithm allows us to connect detritus from a dissipating cloud to its parent cloud, reducing the effective number of small clouds identified in the simulation. Nevertheless, we do not expect our use of the cloud tracking algorithm to significantly alter our results relative to using clouds identified from snapshots of model output.

Cloud core properties of each cloud as a function of height were calculated at each saved time, where cloud core was defined as grid points with condensed liquid water, upward velocity, and positive buoyancy. Cloud core total specific moisture $q_{\mathrm{t}}$ (units of $\mathrm{kg} \mathrm{H}_{2} \mathrm{O}$ per kg moist air), specific condensed liquid water $q_{1}\left(\mathrm{~kg} \mathrm{H}_{2} \mathrm{O}\right.$ per kg moist air), liquid-water potential temperature $\theta_{1}(\mathrm{~K})$, density potential temperature $\theta_{\rho}(\mathrm{K})$, and vertical velocity $w$ profiles were calculated using conditionally sampled horizontal means. Cloud core horizontal area $a$ was found by summing the horizontal area of cloud core grid cells at each height, and cloud core surface area $S\left(\mathrm{~m}^{2}\right)$ was determined by summing the areas of cloud core grid cell faces adjacent to non-core grid cells at each height. Mean horizontal properties for the entire model slab were also recorded to generate cloud anomalies relative to the background mean and mean environmental stratification.

Cloud core buoyancy was calculated as

$B=\frac{g\left(\theta_{\rho}-\overline{\theta_{\rho}}\right)}{\overline{\theta_{\rho}}}$,

where $g\left(\mathrm{~m} \mathrm{~s}^{-2}\right)$ is the acceleration due to gravity, and the bar denotes the horizontal mean over the entire model domain.
Additionally, for each cloud height we calculate the critical mixing fraction $\chi_{\mathrm{c}}$ via de Rooy and Siebesma (2008):

$\chi_{\mathrm{c}}=\frac{\Delta \theta_{\rho}}{\beta \Delta \theta_{\mathrm{l}}+(\beta-\alpha) L /\left(c_{\mathrm{p}} \pi\right) \Delta q_{\mathrm{t}}}$,

where $\Delta \theta_{\rho}=\theta_{\rho}-\overline{\theta_{\rho}}, \Delta \theta_{\mathrm{l}}=\theta_{\mathrm{l}, \mathrm{c}}-\theta_{\mathrm{l}, \mathrm{e}}$ and $\Delta q_{\mathrm{t}}=q_{\mathrm{t}, \mathrm{c}}-q_{\mathrm{t}, \mathrm{e}}$ are the mean cloud-core properties minus the mean properties of the environment, $c_{\mathrm{p}}\left(\mathrm{J} \mathrm{kg}^{-1} \mathrm{~K}^{-1}\right)$ is the specific heat capacity of dry air at constant pressure, $\pi=T / \theta$ is the Exner function, the mean temperature $T(\mathrm{~K})$ of the cloud divided by the mean potential temperature $\theta(\mathrm{K})$, and $\alpha$ and $\beta$ are constants with values of $\alpha \approx 0.12$ and $\beta \approx 0.4$.

Finally, the direct entrainment/detrainment estimation method of Dawe and Austin (2011a) was used to calculate vertical profiles of $\epsilon$ and $\delta$. These calculations were done by horizontally summing the instantaneous mass entrainment $E$ and detrainment $D$ over a region including the cloud core plus all points immediately outside the cloud core. These extra points were included because the tetrahedral interpolation scheme used by Dawe and Austin (2011a) to track the motion of the cloud core surface occasionally locates the surface outside of the cloud core grid cells, which results in entrainment and detrainment occurring outside of the cloud core. This misplacement of the entrainment locations reduces the accuracy of the direct entrainment calculation (which itself is low-biased $\approx 20 \%$ by the interpolation used to generate the cloud core surface) as some mass entrainment and detrainment is displaced vertically. However, the error introduced by this will be random and should not alter the dependence of the entrainment and detrainment rates upon the cloud core properties. The summed $E$ and $D$ values are then divided by the cloud core vertical mass flux $M$ calculated using horizontal cloud core areas calculated by the tetrahedral surface interpolation algorithm to generate self-consistent $\epsilon$ and $\delta$ values (Fig. 1).

This results in 147060 samples of cloud core properties at various heights and times for the BOMEX output, and 134949 samples for the ARM output. Instantaneous cloud samples at a given height consisting of less than 16 grid cells (cross-sectional area $10000 \mathrm{~m}^{2}$ ) were then filtered from the sample set, as they were subject to large amounts of gridscale noise. This mainly has the effect of removing small clouds and the tops and bottoms of larger clouds. Excluding these small area cloud samples removes nearly half of the cloud samples at a given height (Fig. 2a); however, the total cloud fraction (Fig. 2b) and vertical mass flux (Fig. 2c) of the cloud field is only reduced by $\approx 5 \%$. After filtering, 65303 samples remain for the BOMEX output and 87327 samples remain for the ARM output.

\subsection{Cloud core property PDFs}

Here we examine probability density functions (PDFs) of cloud core properties in the BOMEX output. Since the BOMEX case forcing does not vary in time, we amalgamate 
all three hours of model output into a single data set. This results in over 1000 cloud property samples at each height. However, since the decorrelation timescale for individual cloud properties is $\approx 15 \mathrm{~min}$, only $\approx 100$ of these samples are actually independent.

Visual inspection of the distribution of cross-sectional area $a$ of the cloud samples suggests it is consistent with the power law distribution found in studies of shallow cumulus clouds, as the distribution decreases monotonically with size, while total specific water $q_{\mathrm{t}}$, liquid-water potential temperature $\theta_{1}$ and vertical velocity $w$ appear normally distributed (Fig. 3). The mean values of the $q_{\mathrm{t}}, \theta_{\mathrm{l}}$ and $w$ PDFs coincide with the overall horizontal mean values conditionally sampled on the cloud core. The variance of $a$ is relatively constant with height, while the variances of $q_{\mathrm{t}}, \theta_{\mathrm{l}}$ and $w$ steadily increase from cloud base to the start of the inversion at $1500 \mathrm{~m}$. Once in the inversion, the variance of $a, q_{\mathrm{t}}$, and $\theta_{1}$ rapidly decreases with height, while the variance of $w$ remains high.

Next we examine some derived cloud core properties: buoyancy, critical mixing fraction $\chi_{\mathrm{c}}$, and fractional entrainment and detrainment rates (Fig. 4). Buoyancy displays a small positive skewness, and combined with the requirement that $B$ is positive in the cloud core this suggests $B$ is best modelled with a log-normal distribution. Critical mixing fraction shows a normal distribution, while $\epsilon$ and $\delta$ show strong log-normal distributions. The mean values of the cloud core $B$ and $\chi_{\mathrm{c}}$ PDFs again agree with the horizontal mean value of the conditionally sampled core, while the mean of the cloud core $\log _{10}(\epsilon)$ and $\log _{10}(\delta)$ distributions agree with the the $\log _{10}$ of the net cloud core ensemble $\epsilon$ and $\delta$. The variance of $B$ and $\chi_{\mathrm{c}}$ increases through the cloud layer then decreases rapidly in the inversion, while the variances of $\log _{10}(\epsilon)$ and $\log _{10}(\delta)$ are essentially constant with height.

\section{Mutual information analysis}

In this section we analyse merged output from both the ARM and BOMEX cases to determine which cloud core properties are the strongest predictors of the cloud core mass entrainment and detrainment rates. This analysis is complicated by strong correlations between cloud properties (Dawe and Austin, 2012) and non-linear relationships between cloud core variables and entrainment/detrainment rates, which make it difficult to unambiguously link variability in $\epsilon$ and $\delta$ with a single cloud property. In order to overcome these problems, we quantify the strength of dependencies between entrainment/detrainment and cloud properties using the mutual information (MI) shared between them (Shannon and Weaver, 1949).

MI is defined as

$I(X ; Y)=\int P(x, y) \ln \left(\frac{P(x, y)}{P(x) P(y)}\right) \mathrm{d} x \mathrm{~d} y$,
Table 1. Data limits and bin widths used to calculate histograms.

\begin{tabular}{llll}
\hline Variable & Minimum & Maximum & Bin width \\
\hline$z$ & $600 \mathrm{~m}$ & $2600 \mathrm{~m}$ & $100 \mathrm{~m}$ \\
$w$ & $0 \mathrm{~m} \mathrm{~s}^{-1}$ & $6 \mathrm{~m} \mathrm{~s}^{-1}$ & $0.3 \mathrm{~m} \mathrm{~s}^{-1}$ \\
$a$ & $0 \mathrm{~m}^{2}$ & $1.5 \times 10^{6} \mathrm{~m}^{2}$ & $7.5 \times 10^{4} \mathrm{~m}^{2}$ \\
$B$ & $0 \mathrm{~m} \mathrm{~s}^{-2}$ & $5 \times 10^{-2} \mathrm{~m} \mathrm{~s}^{-2}$ & $2.5 \times 10^{-3} \mathrm{~m} \mathrm{~s}^{-2}$ \\
$\chi_{\mathrm{c}}$ & 0 & 0.55 & 0.0275 \\
$\mathrm{~d} \overline{\theta_{\rho}} / \mathrm{d} z$ & $1 \times 10^{-3} \mathrm{~K} \mathrm{~m}^{-1}$ & $0.01 \mathrm{~K} \mathrm{~m}^{-1}$ & $4.5 \times 10^{-4} \mathrm{~K} \mathrm{~m}^{-1}$ \\
$\log _{10}(\epsilon)$ & -3 & -1 & 0.1 \\
$\log _{10}(\delta)$ & -3 & -1 & 0.1 \\
\hline
\end{tabular}

where $P(x), P(y)$, and $P(x, y)$ are the marginal and joint probability density functions for the variables $X$ and $Y$. Similar to the Pearson correlation coefficient, a high MI between two variables implies a strong functional relationship between those variables, but unlike correlation, MI measures non-linear as well as linear relationships. Additional details on the MI calculation are provided in Appendix A.

We estimate the joint PDFs between variables using histograms. PDF estimates generated via histogram are dependant on proper bin choice: too few bins results in a poorlyresolved PDF, while too many bins results in each bin containing too few samples for a reliable PDF estimate. To determine appropriate bin spacing we performed our calculations for a range of bin choices. We restricted the data range so that the majority of bins contained more than 10 samples and found that between 20 and 30 bins generated similar PDFs and MI estimates. All PDFs we present here were calculated with 20 equal-width bins spread across the data range, with the exception of the $\epsilon$ and $\delta$ PDFs which, due to their log-normal distribution, were log-transformed before histogramming. (Repeating our calculations on the un-transformed $\epsilon$ and $\delta$ values gave similar results.) Data limits and bin widths are summarized in Table 1 .

We note here that MI provides a purely statistical analysis of the relationships between variables, without reference to the dynamics of the clouds. The relationships the MI analysis finds have no physical basis and may actually result from indirect correlations between the variables we examine and the true underlying dynamics of the system. The relationships we find may be best considered a kind of null hypothesis: a useful physically-based parameterization of entrainment and detrainment should outperform this statistical analysis. In light of this, we refrain from attempting to interpret our results in dynamical terms until the discussion in Sect. 5 .

\subsection{Entrainment}

In this section we examine the dependence of the fractional mass entrainment rate $\epsilon$ on a variety of cloud variables. The literature provides several examples of entrainment parameterizations using a variety of variable combinations (Turner, 1963; Tiedtke, 1989; Kain and Fritsch, 1990; Neggers et al., 2002; de Rooy and Siebesma, 2008, 2010), but we have 

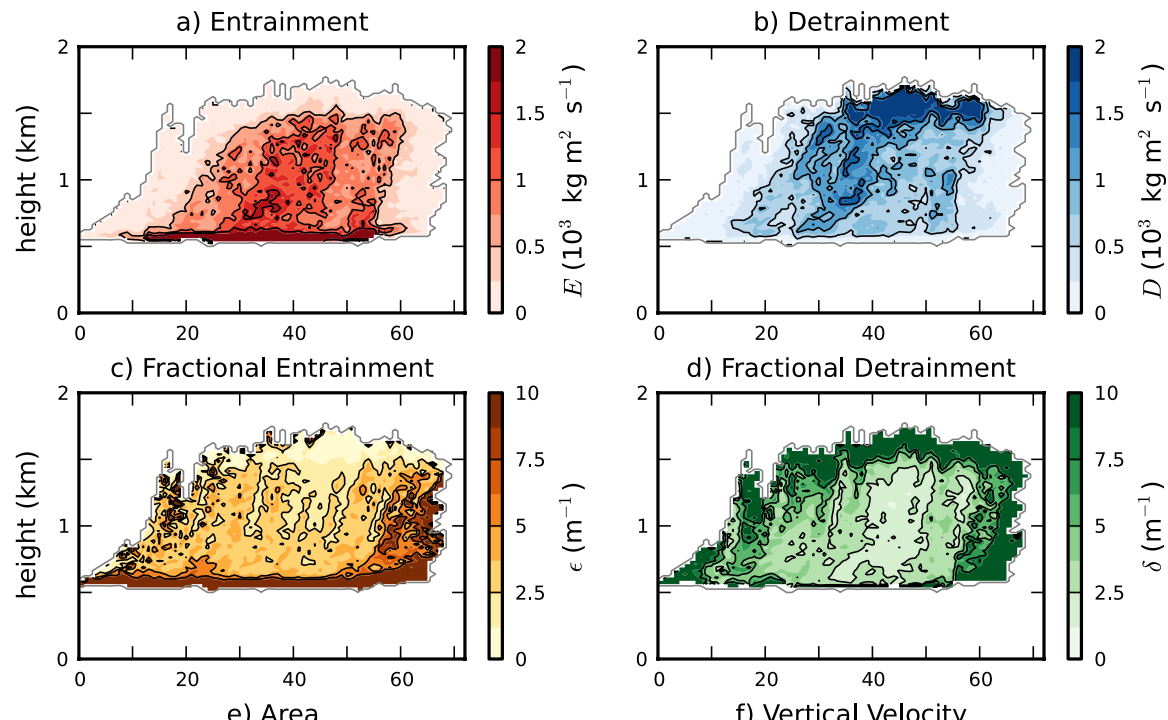

d) Fractional Detrainment
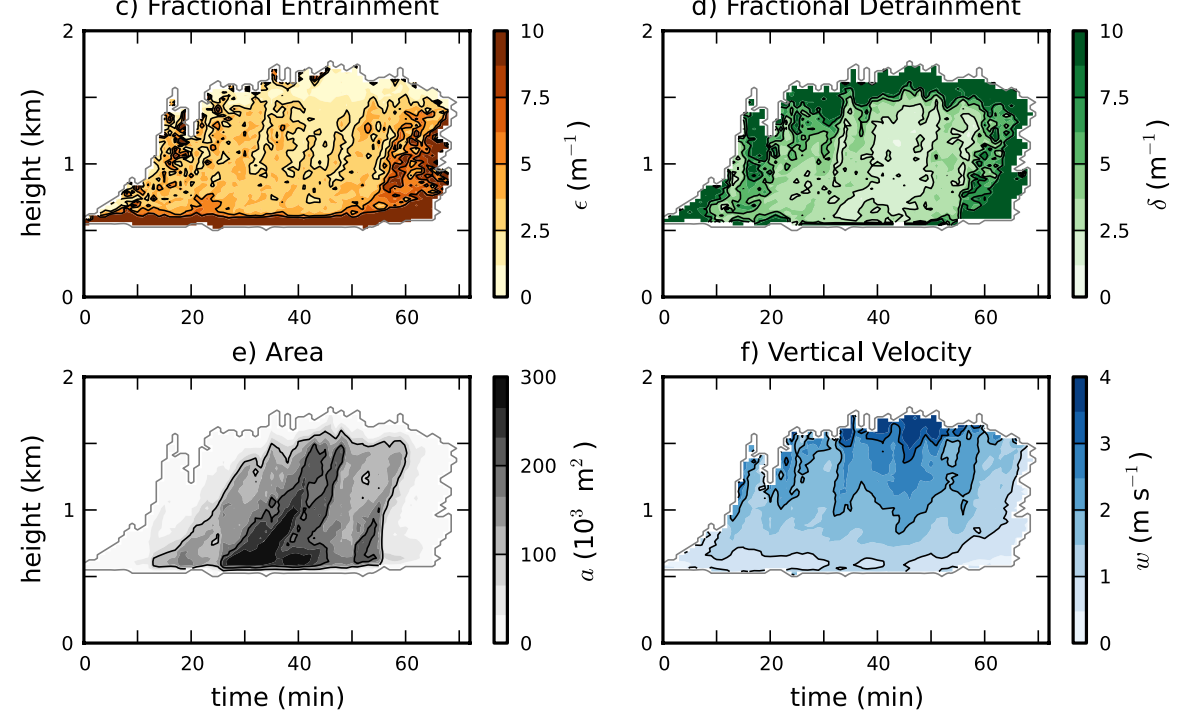

Fig. 1. Height-time profiles of cloud core (a) mass entrainment, (b) mass detrainment, (c) fractional mass entrainment, (d) fractional mass detrainment, (e) cross-sectional area and (f) vertical velocity of the longest-lived tracked cloud in the BOMEX LES output.
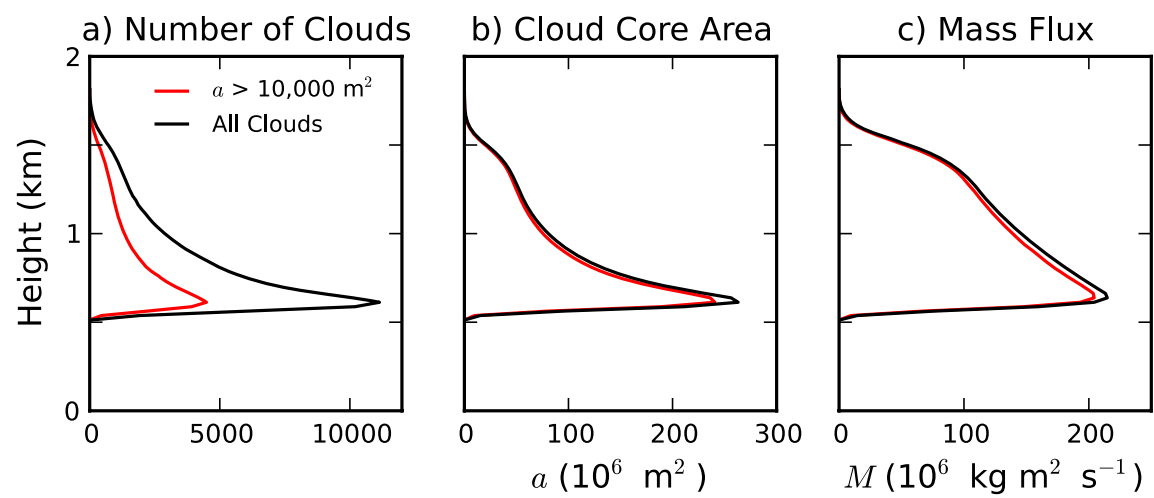

Fig. 2. Vertical profiles of (a) number of cloud core samples, (b) cloud core cross-sectional area, and (c) cloud core vertical mass flux summed over all cloud samples in the entire BOMEX LES run (black line) and all cloud samples with instantaneous cross-sectional area larger than $10000 \mathrm{~m}^{2}$ (red line).

chosen to focus on the basic cloud properties in our analysis for several reasons. First, if the parameterizations have predictive power the MI analysis should pick out the parameterization variables automatically. Second, we perform our calculations using directly measured mass entrainment rates, which differ from the modified rates used in entrainment parameterizations which must account for the influence of the moist cloud shell (Dawe and Austin, 2011b). Third, when we calculated the MI between $\log _{10}(\epsilon)$ and several parameterizations, they generally showed MI values smaller than the cloud variables we present here.

We estimate the joint PDFs between $\log _{10}(\epsilon)$ and the following cloud core properties: vertical velocity $w$, cloud core horizontal area $a$, buoyancy $B$, critical mixing fraction $\chi_{\mathrm{c}}$, 

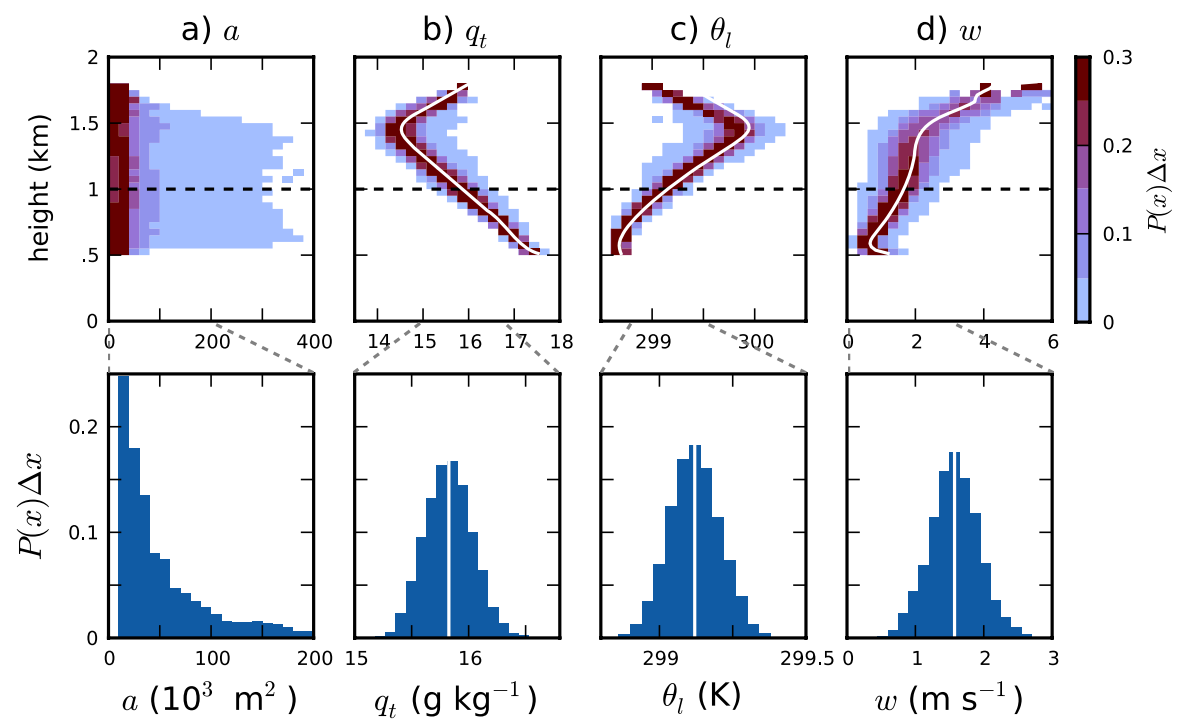

Fig. 3. Probabilities in range $\Delta x(P(x) \Delta x)$ at each model height (top row) and at $1 \mathrm{~km}$ height (bottom row) for cloud core (a) cross-sectional area, (b) total specific humidity, (c) liquid-water potential temperature, and (d) vertical velocity in the BOMEX LES output. White lines indicate the horizontal mean of each variable conditionally sampled on the cloud core over the entire model domain.
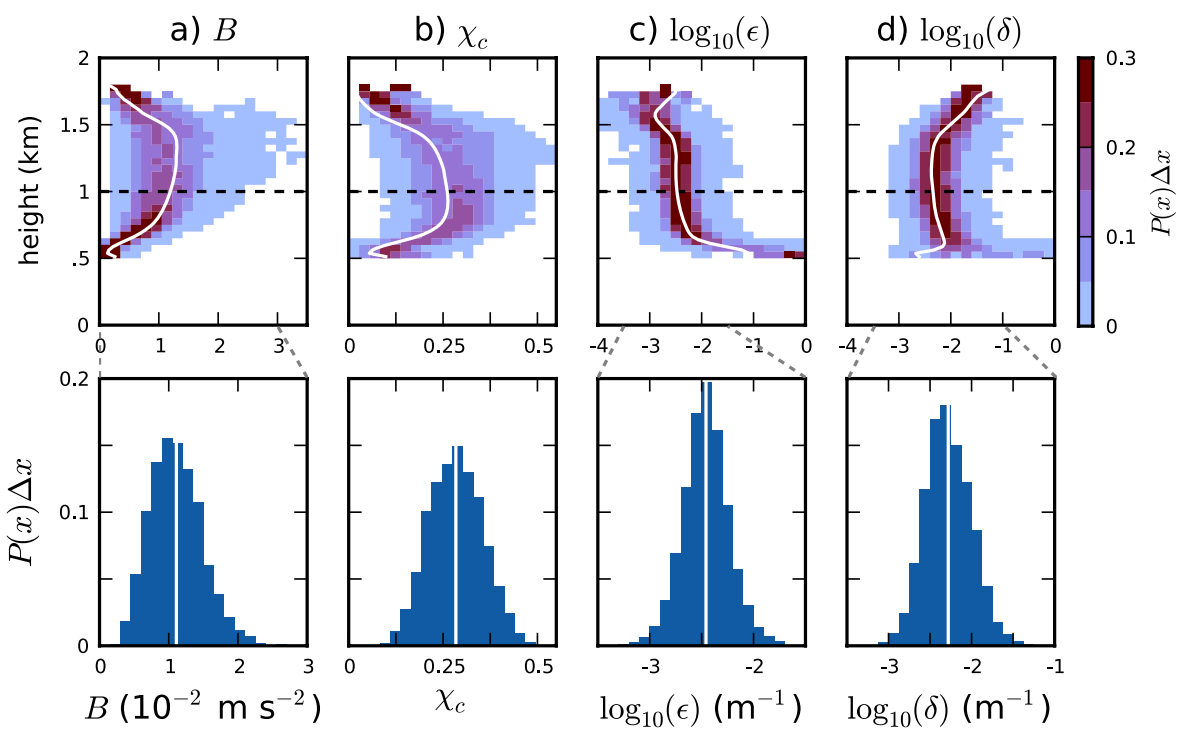

Fig. 4. Probabilities in range $\Delta x(P(x) \Delta x)$ at each model height (top row) and at $1 \mathrm{~km}$ height (bottom row) for cloud core (a) buoyancy, (b) critical mixing fraction, (c) $\log _{10}$ of the fractional mass entrainment, and (d) $\log _{10}$ of the fractional mass detrainment in the BOMEX LES output. White lines indicate the horizontal mean of each variable conditionally sampled on the cloud core over the entire model domain.

the lapse rate of environmental density potential temperature $\mathrm{d} \theta_{\rho} / \mathrm{d} z\left(\mathrm{~K} \mathrm{~m}^{-1}\right)$, and the height $z(\mathrm{~m})$. We consider the joint PDF of $\log _{10}(\epsilon)$ and height $z$ as a null hypothesis, as there is little reason the absolute height above ground should, by itself, affect the entrainment rate. The resulting joint PDFs display remarkably similar behaviour for all variables, with larger variable values associated with smaller $\log _{10}(\epsilon)$ (Fig. 5). This is not surprising in light of the strong correlations present between shallow cumulus cloud properties (Dawe and Austin, 2012).

MI values for $\log _{10}(\epsilon)$ are given in Table 2. Buoyancy $B$ shows the largest MI value with $\log _{10}(\epsilon)$, with a value nearly double the next largest, $I\left(\log _{10}(\epsilon) ; \chi_{\mathrm{c}}\right)$. All variables show MI values larger than the maximum value generated by calculating the MI between $\log _{10}(\epsilon)$ and 100 random permutations of each variable, which we use as a measurement 


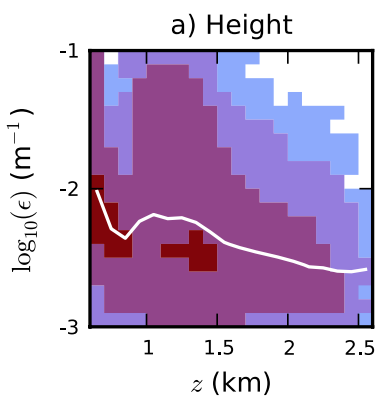

d) Buoyancy

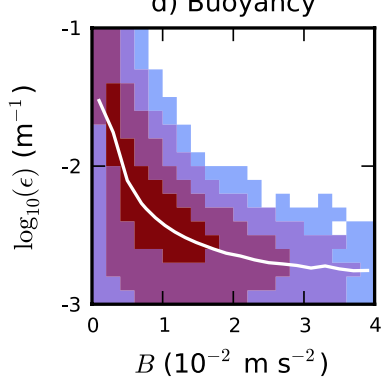

b) Vertical Velocity

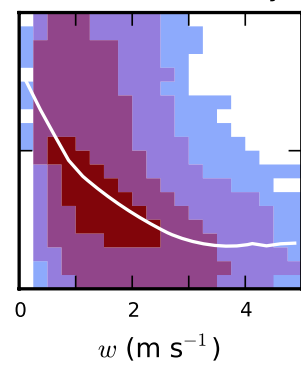

e) Crit. Mixing Frac.

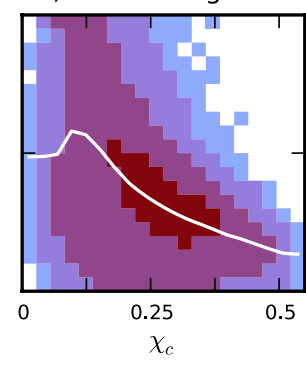

c) Area

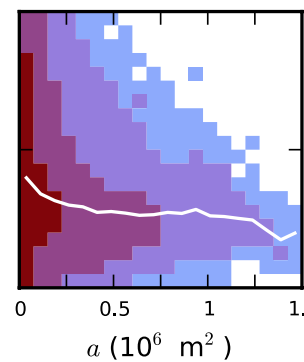

f) Stratification

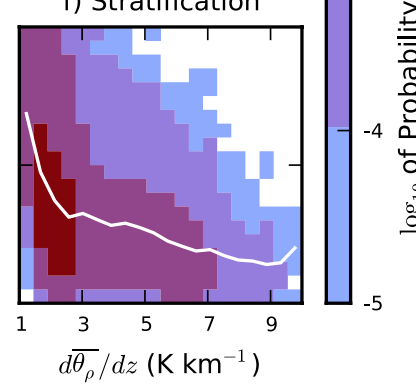

Fig. 5. Joint probability density functions multiplied by bin area $(P(x, y) \Delta x \Delta y)$ for individual clouds in the combined BOMEX and ARM LES output of $\log _{10}(\epsilon)$ versus (a) height, (b) vertical velocity, (c) cross-sectional area, (d) buoyancy, (e) critical mixing fraction, and (f) vertical gradient of environmental density potential temperature. PDFs are plotted using a logarithmic scale. White lines indicate the mean $\log _{10}(\epsilon)$ value as a function of the $x$ axis variable.

of statistical significance. However, $I\left(\log _{10}(\epsilon) ; a\right)$ is smaller than $I\left(\log _{10}(\epsilon) ; z\right)$, suggesting $a$ has little influence on the entrainment rate.

Cross-sectional area $a$ shows the smallest MI value with $\log _{10}(\epsilon)$, and the relative lack of dependence of the mean value of $\log _{10}(\epsilon)$ on $a$ is readily apparent in the PDF (Fig. 5c). This is surprising, as we would expect entrainment rate to be related to the surface area of the core surface, which in turn should be related to the area occupied by the clouds. However, the variance in $\log _{10}(\epsilon)$ is strongly dependent on $a$, with the largest and smallest values of $\log _{10}(\epsilon)$ only occurring for the smallest area clouds. We take this to indicate a strong patchiness and spatial localization in the distribution of entrainment. Small clouds may be subject to flow structures that drive large or small amounts of entrainment, but large clouds average over these flow structures, mitigating the variability in entrainment they experience. Nevertheless, even at the largest cloud sizes, there is still nearly an order of magnitude range in the variability of $\epsilon$.

One possible cause of the relative constancy of $\log _{10}(\epsilon)$ versus $a$ is the existence of correlations between $a$ and other cloud core properties. For example, cloud core area is positively correlated with buoyancy (Dawe and Austin, 2012). If larger area clouds tended to have reduced $\log _{10}(\epsilon)$ this would be offset in the joint PDFs by the tendency for high buoyancies to increase $\log _{10}(\epsilon)$; the true dependence of $\log _{10}(\epsilon)$ on $a$ would be masked by the covariance of $a$ and $B$.
Table 2. Mutual information between $\log _{10}(\epsilon)$ and various cloud core properties for individual clouds in the combined BOMEX and ARM LES output. Noise level is found by taking the maximum of 100 Monte Carlo trials of mutual information between $\log _{10}(\epsilon)$ and a random permutation of each variable. Maximum mutual information values in each comparison category are in bold.

\begin{tabular}{lrc}
\hline Variable & MI & Noise \\
\hline$I\left(\log _{10}(\epsilon) ; z\right)$ & 0.109 & 0.002 \\
$I\left(\log _{10}(\epsilon) ; w\right)$ & 0.221 & \\
$I\left(\log _{10}(\epsilon) ; a\right)$ & 0.038 & \\
$I\left(\log _{10}(\boldsymbol{\epsilon}) ; \boldsymbol{B}\right)$ & $\mathbf{0 . 4 3 0}$ & \\
$I\left(\log _{10}(\epsilon) ; \chi_{\mathrm{c}}\right)$ & 0.261 & \\
$I\left(\log _{10}(\epsilon) ; \mathrm{d} \bar{\theta}_{\rho} / \mathrm{d} z\right)$ & 0.127 & \\
\hline$I\left(\log _{10}(\epsilon) ; z \mid B\right)$ & 0.07 & 0.01 \\
$I\left(\log _{10}(\epsilon) ; w \mid B\right)$ & 0.06 & \\
$I\left(\log _{10}(\epsilon) ; a \mid B\right)$ & 0.03 & \\
$I\left(\log _{10}(\epsilon) ; \chi_{\mathrm{c}} \mid B\right)$ & 0.07 & \\
$I\left(\log _{10}(\boldsymbol{\epsilon}) ; \mathbf{d} \bar{\theta} / \mathbf{d} z \mid \boldsymbol{B}\right)$ & $\mathbf{0 . 1 3}$ & \\
\hline$I\left(\log _{10}(\epsilon) ; z \mid B, \mathrm{~d} \overline{\theta_{\rho}} / \mathrm{d} z\right)$ & 0.13 & 0.10 \\
$I\left(\log _{10}(\epsilon) ; w \mid B, \mathrm{~d} \overline{\theta_{\rho}} / \mathrm{d} z\right)$ & 0.09 & 0.08 \\
$I\left(\log _{10}(\epsilon) ; a \mid B, \mathrm{~d} \overline{\theta_{\rho}} / \mathrm{d} z\right)$ & 0.08 & 0.06 \\
$I\left(\log _{10}(\epsilon) ; \chi_{\mathrm{c}} \mid B, \mathrm{~d} \overline{\theta_{\rho}} / \mathrm{d} z\right)$ & 0.11 & 0.10 \\
\hline
\end{tabular}

We can separate out the effects of these correlations by generating joint PDFs of $\log _{10}(\epsilon)$ with two variables simultaneously. To do this we calculate three dimensional 
histograms to estimate $P\left(\log _{10}(\epsilon), y, \zeta\right)$, where $y$ and $\zeta$ are various combinations of the cloud core properties $z, a, B$, $w$, and $\mathrm{d} \overline{\theta_{\rho}} / \mathrm{d} z$. (We use $\zeta$ for the third PDF variable as we have already designated $z$ to represent height.) The resulting histograms show little change when calculated using 20 and 30 bins along each dimension, so we maintain the same bin widths as used in generating the two dimensional histograms.

These three dimensional joint PDFs reveal a great deal of information about the behaviour of $\epsilon$, but can be difficult to visualize in two dimensions. Visual inspection of the distributions of $\log _{10}(\epsilon)$ at various points in the $(y, \zeta)$ space show reasonably Gaussian distributions. If $\epsilon$ is log-normally distributed at all points in the $(y, \zeta)$ space, then half of the $\epsilon$ distribution should have larger values than the mean of $\log _{10}(\epsilon)$ and half should have smaller values. Thus, to visualize these PDFs we plot the mean of $\log _{10}(\epsilon)$ over the $(y, \zeta)$ space to show how the distributions change with variables.

The easiest plot to interpret is probably the mean of the joint PDF of $\log _{10}(\epsilon)$, height, and area $P\left(\log _{10}(\epsilon), z, a\right)$ (Fig. 6, row 1, column 1), which clearly shows the vertical variation in $\epsilon$ with height and the slight decrease in $\epsilon$ as cloud area increases. Since we do not expect height to directly influence $\epsilon$, apparent variations in $\epsilon$ with height actually arise due to changes in the mean cloud properties. This is apparent comparing $P\left(\log _{10}(\epsilon), z, a\right)$ to $P\left(\log _{10}(\epsilon), z, B\right)$ (Fig. 6, row 1 , column 2), in which nearly all the variation in $\epsilon$ collapses onto changes in $B$. In fact, at nearly every height the mean of $\epsilon$ is better correlated with $B, \chi_{\mathrm{c}}, w$ and $\mathrm{d} \bar{\theta}_{\rho} / \mathrm{d} z$ than $z$ (Fig. 6, row 1). Similarly, the mean of the joint PDFs of $\log _{10}(\epsilon)$ and $a$ (Fig. 6, column 1) show the apparent variation of $\epsilon$ with $a$ is better explained by correlations between $a$ and other cloud properties.

The remaining plots are less clear-cut, with buoyancy, critical mixing fraction, and vertical velocity all displaying strong independent covariability with $\epsilon$. Buoyancy shows the strongest covariance with $\epsilon$ (Fig. 6, column 2), in agreement with the MI calculations, but it is difficult to judge which variable is the second most important. To quantify which variable provides the most information about $\epsilon$ that is independent of $B$, we calculate the conditional mutual information (CMI) for each PDF:

$$
I(X ; Y \mid Z)=\int P(x, y, \zeta) \ln \left(\frac{P(x, y \mid \zeta)}{P(x) P(y \mid \zeta)}\right) \mathrm{d} x \mathrm{~d} y \mathrm{~d} \zeta
$$

(We have designated samples of the random variable $Z$ with $\zeta$ to avoid confusion with the height $z$.) By conditioning the PDF of $Y$ on the value of $Z$, CMI removes the mutual information between $X$ and $Z$, revealing the MI between $X$ and $Y$.

We calculate CMI between $\log _{10}(\epsilon)$ and the cloud core properties conditioned on $B$ to determine which variable provides the most information that is not already provided by $B$ (Table 2). $\mathrm{d} \overline{\theta_{\rho}} / \mathrm{d} z$ shows the largest CMI with $\log _{10}(\epsilon)$ when conditioned on $B$, despite the small MI between $\log _{10}(\epsilon)$ and $\mathrm{d} \overline{\theta_{\rho}} / \mathrm{d} z$. Note that the CMI between $\log _{10}(\epsilon)$
Table 3. Mutual information between $\log _{10}(\delta)$ and various cloud core properties for individual clouds in the combined BOMEX and ARM LES output. Noise level is found by taking the maximum of 100 Monte Carlo trials of mutual information between $\log _{10}(\delta)$ and a random permutation of each variable. Maximum mutual information values in each comparison category are in bold.

\begin{tabular}{lrc}
\hline Variable & MI & Noise \\
\hline$I\left(\log _{10}(\delta) ; z\right)$ & 0.026 & 0.002 \\
$I\left(\log _{10}(\delta) ; w\right)$ & 0.218 & \\
$I\left(\log _{10}(\delta) ; a\right)$ & 0.159 & \\
$I\left(\log _{10}(\delta) ; B\right)$ & 0.184 & \\
$I\left(\log _{10}(\delta) ; \chi_{\mathbf{c}}\right)$ & $\mathbf{0 . 3 4 5}$ & \\
$I\left(\log _{10}(\delta) ; \mathrm{d} \overline{\theta_{\rho}} / \mathrm{d} z\right)$ & 0.058 & \\
\hline$I\left(\log _{10}(\delta) ; z \mid \chi_{\mathrm{c}}\right)$ & 0.04 & 0.02 \\
$I\left(\log _{10}(\delta) ; w \mid \chi_{\mathbf{c}}\right)$ & $\mathbf{0 . 1 7}$ & \\
$I\left(\log _{10}(\delta) ; a \mid \chi_{\mathrm{c}}\right)$ & 0.08 & \\
$I\left(\log _{10}(\delta) ; B \mid \chi_{\mathrm{c}}\right)$ & 0.03 & \\
$I\left(\log _{10}(\delta) ; \mathrm{d} \overline{\theta_{\rho}} / \mathrm{d} z \mid \chi_{\mathrm{c}}\right)$ & 0.04 & \\
\hline$I\left(\log _{10}(\delta) ; z \mid \chi_{\mathrm{c}}, w\right)$ & 0.27 & 0.13 \\
$I\left(\log _{10}(\delta) ; a \mid \chi_{\mathrm{c}}, w\right)$ & 0.10 & 0.07 \\
$I\left(\log _{10}(\delta) ; B \mid \chi_{\mathrm{c}}, w\right)$ & 0.12 & 0.08 \\
$I\left(\log _{10}(\delta) ; \mathrm{d} \overline{\theta_{\rho}} / \mathrm{d} z \mid \chi_{\mathrm{c}}, w\right)$ & 0.21 & 0.09 \\
\hline
\end{tabular}

and $\mathrm{d} \overline{\theta_{\rho}} / \mathrm{d} z$ conditioned on $B$ is higher than the MI between $\log _{10}(\epsilon)$ and $\mathrm{d} \overline{\theta_{\rho}} / \mathrm{d} z$, indicating that correlations between $B$ and $\mathrm{d} \overline{\theta_{\rho}} / \mathrm{d} z$ were obscuring the true strength of the dependence of $\epsilon$ on $\mathrm{d} \overline{\theta_{\rho}} / \mathrm{d} z$. (Clouds in strong stratification tend to have low buoyancy, which increases $\epsilon$.) Examination of $P\left(\log _{10}(\epsilon), \mathrm{d} \overline{\theta_{\rho}} / \mathrm{d} z, B\right)$ (Fig. 6, row 2, column 2) shows the largest $\epsilon$ values are present at low buoyancy and stratification.

Calculating the remaining CMI of $\log _{10}(\epsilon)$ conditioned on both $B$ and $\mathrm{d} \overline{\theta_{\rho}} / \mathrm{d} z$ shows values close to the noise level of the calculation (Table 2). This indicates that nearly all the information about $\epsilon$ recoverable from the cloud core state can be found using only $B$ and $\mathrm{d} \overline{\theta_{\rho}} / \mathrm{d} z$. Note that this may be an artifact of an insufficient number of samples to properly resolve the full multi-dimensional histograms.

\subsection{Detrainment}

In this section we repeat the previous analysis to examine the dependence of the fractional mass detrainment rate $\delta$ on the cloud core properties. The joint PDFs of $\log _{10}(\delta)$ with the cloud properties (Fig. 7) are a little more complex than the $\log _{10}(\epsilon)$ PDFs. As with the entrainment, larger $w, a, B$, and $\chi_{\mathrm{c}}$ values are associated with smaller $\log _{10}(\delta)$. Unlike the entrainment, $\log _{10}(\delta)$ appears to increase with stronger stratification when $\mathrm{d} \theta_{\rho} / \mathrm{d} z \geq 3 \mathrm{~K} \mathrm{~km}^{-1}$. Detrainment shows slightly more dependence on $a$ than entrainment, though this covariance is still small relative to the other variables. Finally, $\log _{10}(\delta)$ decreases with $w$ between $0-3 \mathrm{~m} \mathrm{~s}^{-1}$ but increases between $3-6 \mathrm{~m} \mathrm{~s}^{-1}$. 

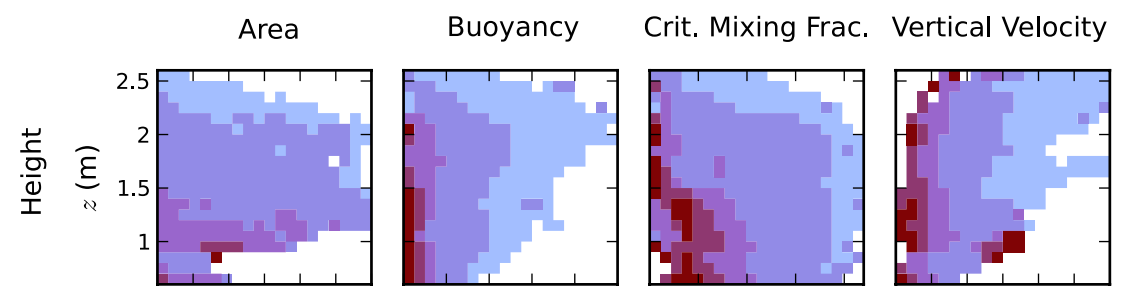

Stratification
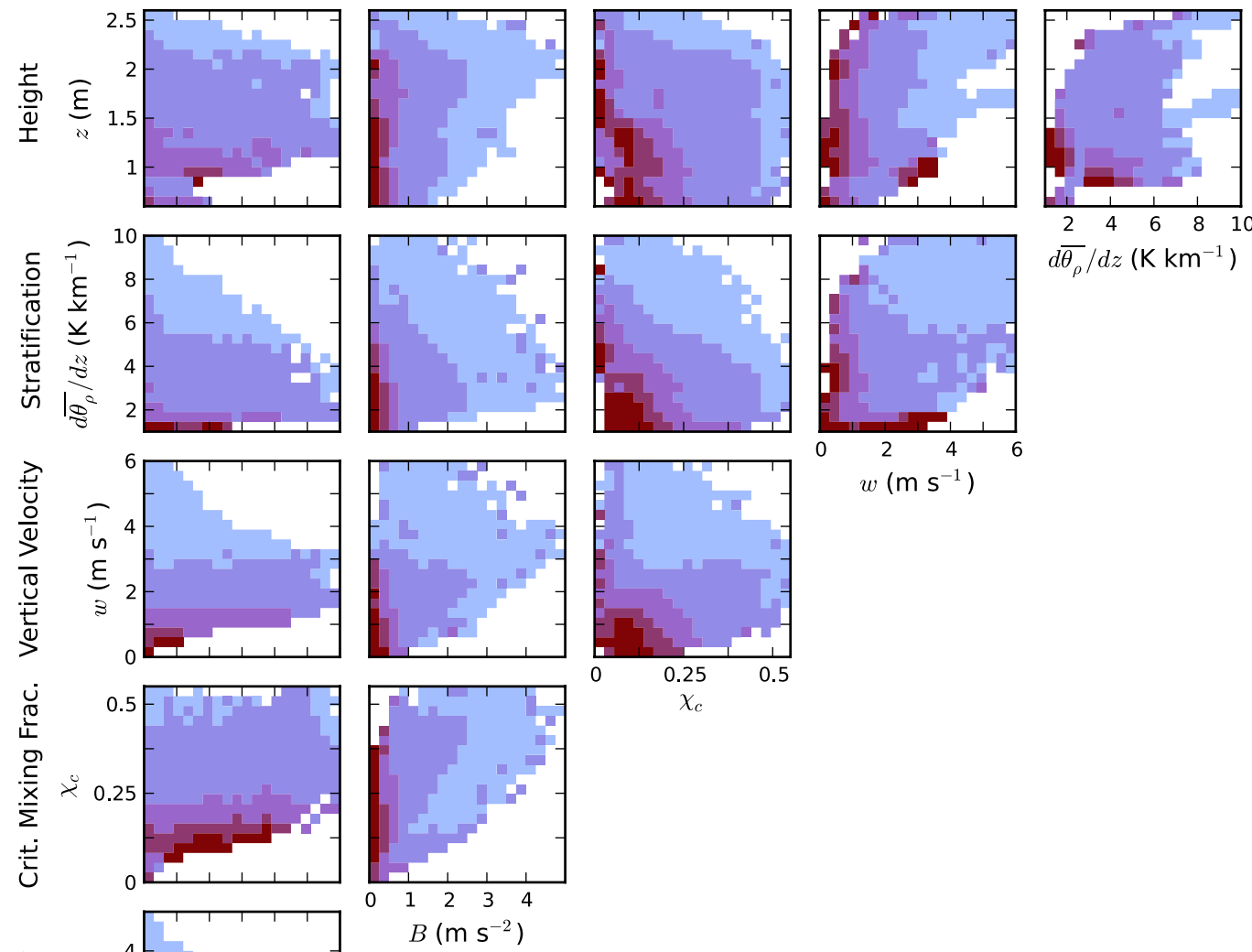

$w\left(\mathrm{~m} \mathrm{~s}^{-1}\right)$
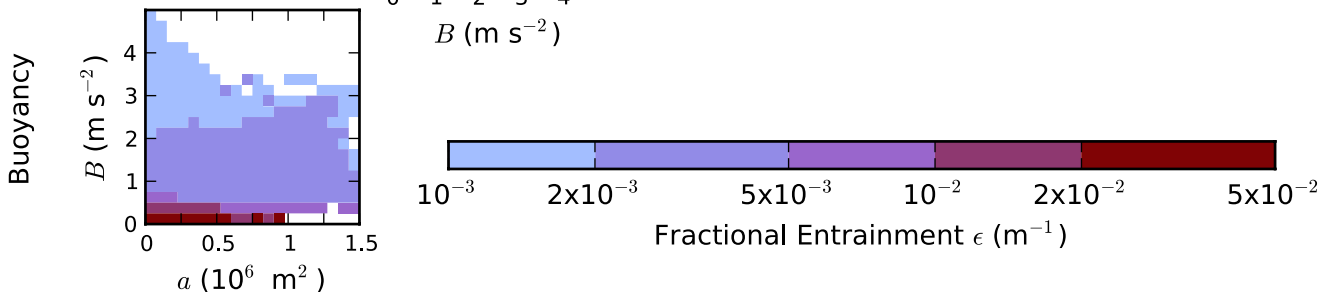

Fig. 6. Mean values of $\log _{10}(\epsilon)$ for each bin of joint probability density functions of various cloud core properties for individual clouds in the combined BOMEX and ARM LES output. The $y$ axis of each row shows height, environmental stratification, critical mixing fraction, buoyancy, and vertical velocity (from top to bottom), and the $x$ axis of each column shows cross-sectional area, vertical velocity, buoyancy, critical mixing fraction, and environmental stratification (from left to right).

Values of MI between $\log _{10}(\delta)$ and the cloud properties are given in Table 3. The largest MI value results from $I\left(\log _{10}(\delta) ; \chi_{\mathrm{c}}\right)$, implying that the critical mixing fraction is the best predictor of detrainment rate. This result is in broad agreement with a range of previous work on parameterization of cloud core detrainment (Kain and Fritsch, 1990; de Rooy and Siebesma, 2008; Bretherton and Park, 2009).

Mean values of joint PDFs for $\log _{10}(\delta)$ are presented in Fig. 8. These clearly display the strong relationship between $\delta$ and $\chi_{\mathrm{c}}$. The relatively strong dependence of $\log _{10}(\delta)$ on buoyancy disappears completely when $P\left(\log _{10}(\delta), \chi_{\mathrm{c}}, B\right)$ (Fig. 8, row 4, column 2) is examined. Area $a$ shows a moderate effect on $\log _{10}(\delta)$ that is independent of $\chi_{\mathrm{c}}$, but the vertical velocity $w$ shows the largest effect on $\log _{10}(\delta)$ independent of $\chi_{\mathrm{c}}$ (Fig. 8, row 3, column 3). The largest detrainment rates occur when both $\chi_{\mathrm{c}}$ and $w$ are small. This is confirmed by calculating CMI values between $\log _{10}(\delta)$ and the cloud core properties conditioned on $\chi_{\mathrm{c}}$ (Table 3 ). The vertical velocity shows the largest CMI with $\log _{10}(\delta)$, over twice the CMI of $a$ with $\log _{10}(\delta)$ conditioned on $\chi_{\mathrm{c}}$.

This strong inverse relationship between $\log _{10}(\delta)$ and $w$ is reminiscent of the parameterization of Neggers et al. (2002). Neggers et al. proposed a $w^{-1}$ behaviour for $\epsilon$, not $\delta$, but it is not implausible that turbulent entrainment and detrainment would follow the same behaviour. Furthermore, since $w$ and $B$ are correlated (Dawe and Austin, 2012), a dependence of $\epsilon$ on $B$ would also cause a correlation between $\epsilon$ and $w$. As our analysis is purely statistical we are unable to unambiguously attribute the behaviour of $\epsilon$ and $\delta$ to dependence on $B$ or $w$, 

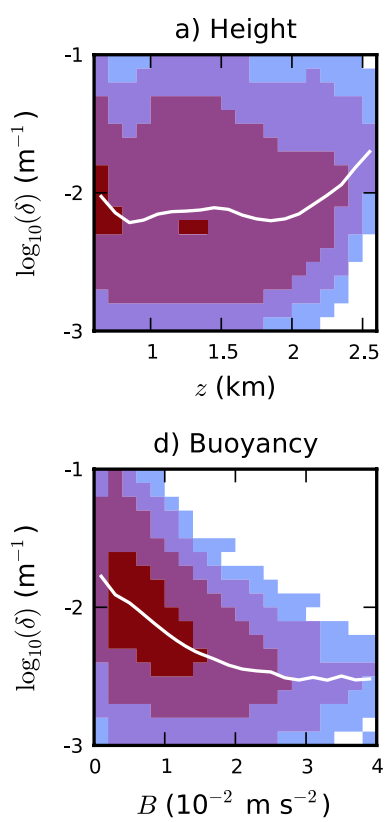

b) Vertical Velocity

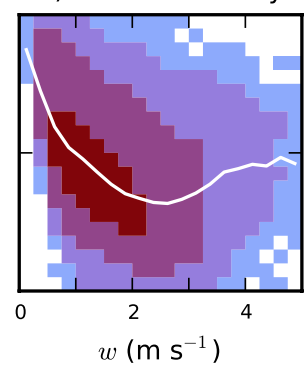

e) Crit. Mixing Frac.

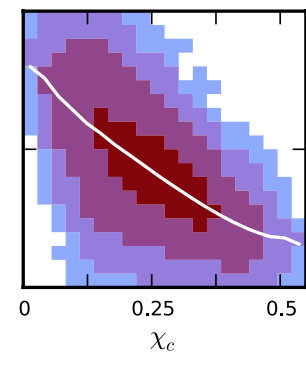

c) Area

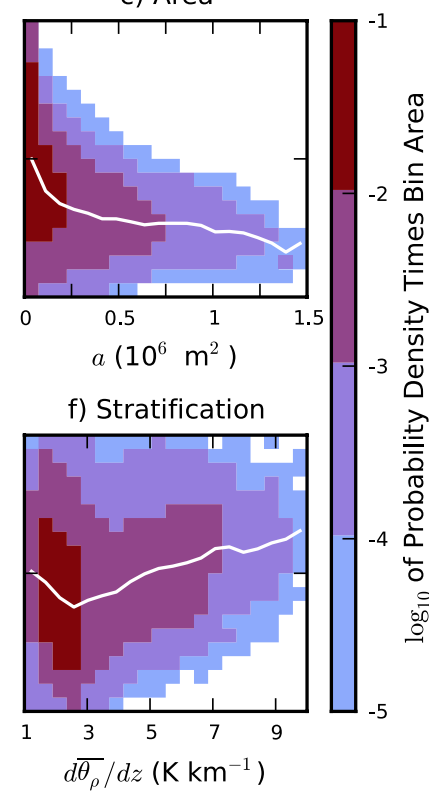

Fig. 7. Joint probability density functions multiplied by bin area $(P(x, y) \Delta x \Delta y)$ for individual clouds in the combined BOMEX and ARM LES output of $\log _{10}(\delta)$ versus (a) height, (b) vertical velocity, (c) cross-sectional area, (d) buoyancy, (e) critical mixing fraction, and (f) vertical gradient of environmental density potential temperature. PDFs are plotted using a logarithmic scale. White lines indicate the mean $\log _{10}(\delta)$ value as a function of the $x$ axis variable.

but either way, these results support the constant timescale $w^{-1}$ behaviour observed by Neggers et al.

Finally, we calculate CMI values of $\log _{10}(\delta)$ conditioned on both $\chi_{\mathrm{c}}$ and $w$ (Table 3). The largest CMI value in this case results from $z$, and is only roughly twice the statistical noise level, so we conclude there is little meaningful information remaining.

\subsection{Cloud perimeter vs. area}

An interesting result of the previous analysis is the apparent independence of $\epsilon$ and cloud cross-sectional area $a$. Many entrainment parameterizations follow the assumption made by Turner (1963) that entrainment follows the scaling

$$
E=k \rho w C=2 k \rho w \frac{a}{R},
$$

where $k$ is a dimensionless constant, $C$ is the perimeter of the cloud cross section (m), and $R$ is the cloud radius (m) (Arakawa and Schubert, 1974; Kain and Fritsch, 1990). (The second form of the equation is derived by assuming the cloud is cylindrical, so $C=2 \pi R$ and $a=\pi R^{2}$.) This assumption appears to be incorrect; fractional entrainment rate is almost independent of cloud area, at least for shallow cumulus. This may help explain the efficacy of the assumption made by some parameterizations (Tiedtke, 1989; Bretherton and Park, 2009) that $R$ is constant, which implies $C \propto a, E \propto \rho w a$ and thus $\epsilon=E / M$ is independent of area.
Real clouds, of course, are not cylindrical. If the perimeter of the fractal cloud surface were to scale linearly with $a$, this would explain the relatively constant value of $\epsilon$ with $a$. Siebesma and Jonker (2000) found a $C \propto a^{0.66}$ relationship in a BOMEX LES where $a$ and $C$ were calculated from a two-dimensional projection of the cloud area to mimic satellite observations, significantly different from the $C \propto$ $a^{0.5}$ relationship one would expect for a cylindrical cloud. However, entrainment will depend on the area-perimeter relationship of horizontal cross sections through the cloud, which may differ from the two-dimensional projection used in their study.

In light of this, we calculate the perimeter-area relationship for horizontal cloud slices in our LES output. Since the LES is a discrete model, we calculate a pseudo-perimeter for each cloud by taking the cloud surface area at a given height and dividing it by the LES vertical grid spacing $\mathrm{d} z(25 \mathrm{~m})$. We calculate a fit of the curve $C=k a^{n}$ to the data by performing a linear least-squares best fit between $\log a$ and $\log C$ to find $\log C=n \log a+\log (k)$, which results in $n=0.73$ and $k=1.50\left(C=1.50 a^{0.73}\right.$, correlation $0.950 \pm 0.001$, root mean square (RMS) error $2158 \mathrm{~m}$, Fig. 9). This relationship shows a significantly larger correlation than either a linear $(C=0.043 a$, correlation $0.934 \pm 0.001$, RMS error $3008 \mathrm{~m})$ or a square root $(C=26.0 \sqrt{a}$, correlation $0.945 \pm 0.001$, RMS error $2695 \mathrm{~m}$ ) relationship between $C$ and $a$, where we have constrained these fits so that $C(0)=0$. 

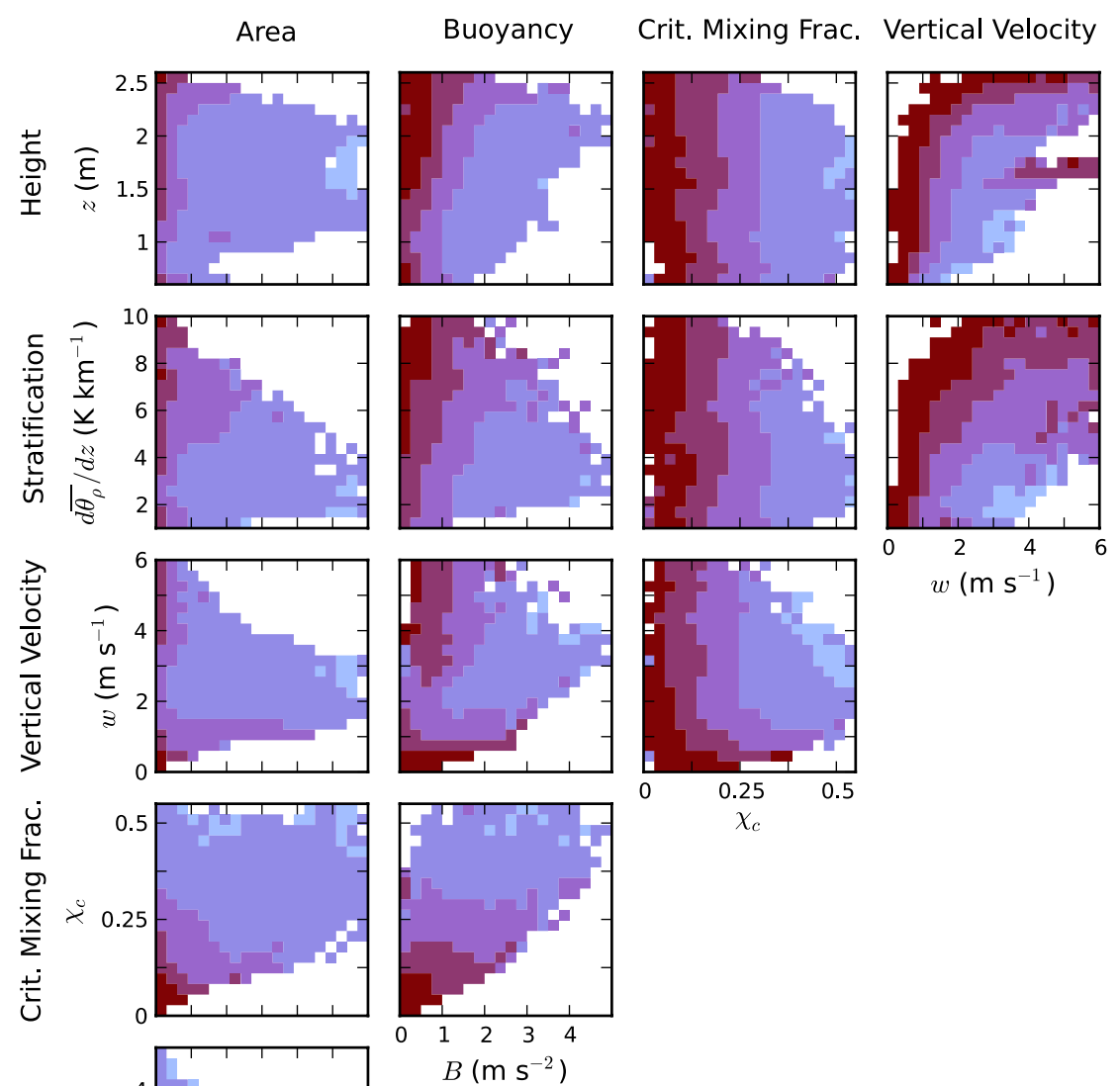

Stratification

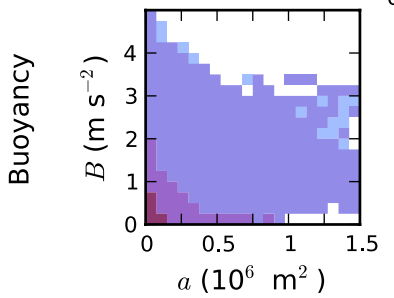

$B\left(\mathrm{~m} \mathrm{~s}^{-2}\right)$

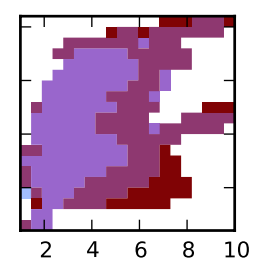

$d \overline{\theta_{\rho}} / d z\left(\mathrm{~K} \mathrm{~km}^{-1}\right)$

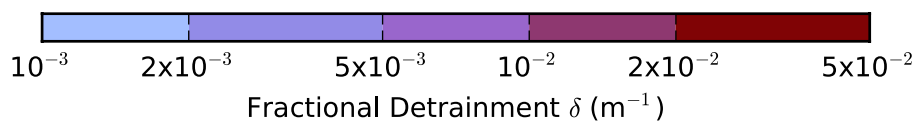

Fig. 8. Mean values of $\log _{10}(\delta)$ for each bin of joint probability density functions for various cloud core properties of individual clouds in the combined BOMEX and ARM LES output. The $y$ axis of each row shows height, environmental stratification, critical mixing fraction, buoyancy, and vertical velocity (from top to bottom), and the $x$ axis of each column shows cross-sectional area, vertical velocity, buoyancy, critical mixing fraction, and environmental stratification (from left to right).

If $C \propto a^{0.73}$, then Eq. (7) implies $\epsilon \propto a^{-0.27}$. However, our analysis also shows that $\epsilon$ is independent of $a$ when other variables are held constant. This contradiction implies that the basic concept underlying Eq. (7) - that mass entrainment flux $E$ is proportional to the cloud surface area - is not true for these simulated clouds.

We can check this using the LES output by fitting power law relationships between the entrainment and detrainment fluxes and the cloud core area and pseudo-perimeter. Doing so shows $E \propto a^{0.97 \pm 0.01}, E \propto C^{1.29 \pm 0.01}, D \propto a^{0.87 \pm 0.01}$, and $D \propto C^{1.08 \pm 0.01}$ (Fig. 9). Thus, it appears that $E$ is indeed proportional to cross-sectional area, while $D$ is not obviously proportional to either $a$ or $C$.

Why this surprising result should be the case is not readily apparent. We find approximately the same results when we filter cloud heights within $100 \mathrm{~m}$ of cloud base, where
$E$ might reasonably be expected to be proportional to crosssectional area due to the condensation-produced buoyancy of the rising thermals. In any case, the linear dependence between $E$ and $a$ is clearly fortuitous for the purposes of cloud parameterizations.

\section{Parameterization of entrainment and detrainment rates}

While mutual information provides us with a way to measure the dependencies between variables in a data set, it says nothing about the functional form of those dependencies. In this section we attempt to construct a parameterization for the $\epsilon$ and $\delta$ of individual shallow cumulus clouds by curve fitting simple power law relationships for $\epsilon\left(B, \mathrm{~d} \overline{\theta_{\rho}} / \mathrm{d} z\right)$ and 


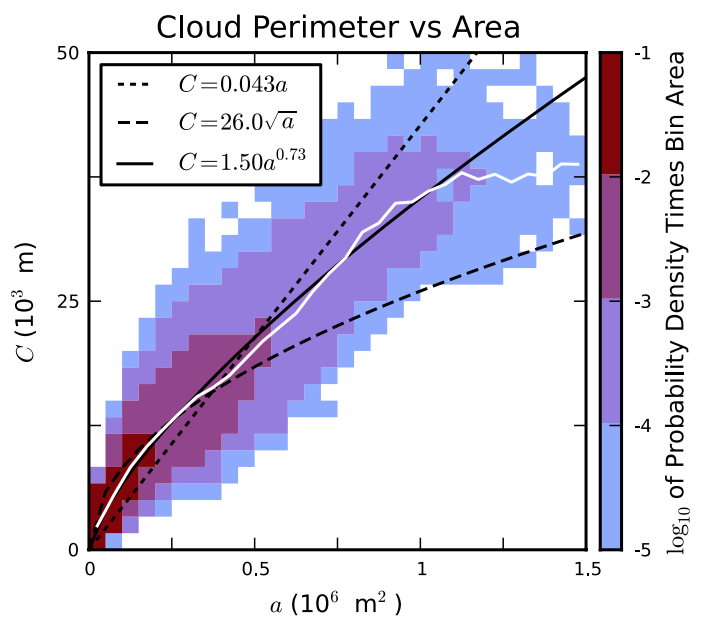

Fig. 9. Joint probability density function multiplied by bin area of cloud core perimeter $C$ versus cloud core cross-sectional area $a$ $(P(a, C) \Delta a \Delta C)$ for individual clouds in the combined BOMEX and ARM LES output. PDF is plotted using a logarithmic scale. White line shows the mean perimeter of clouds as a function of cross-sectional area. Black dotted, dashed, and solid lines show best-fit lines for linear, square root, and an arbitrary power law relationships, respectively.

$\delta\left(w, \chi_{c}\right)$. Additionally, we attempt to extend these fits to parameterize $\epsilon$ and $\delta$ values for the overall cloud ensemble.

We wish to emphasize that these parameterizations are purely statistical in nature, with little reference to the underlying dynamics of the system. For example, they do not produce relationships with units of $\mathrm{m}^{-1}$ and require constant multipliers with units that correct for dimensional consistency, unlike most published parameterizations. We do not advocate that statistical fits be used as parameterizations without an understanding of the dynamics of the system, but instead we suggest they be used as a null hypothesis for the behaviour of $\epsilon$ and $\delta$. In other, words, parameterizations of shallow cumulus mass entrainment and detrainment should at minimum display higher correlation and lower RMS error when compared with statistical power law fits to be considered valid. However, since most currently published parameterizations predict tracer, not mass, entrainment rates, we are not able to directly compare our results.

\subsection{Entrainment}

In this section we examine the dependence of fractional entrainment $\epsilon$ on buoyancy $B$ and stratification $\mathrm{d} \overline{\theta_{\rho}} / \mathrm{d} z$. We fit power law relationships between the variables by performing linear least-squares best fits between $\log _{10}(\epsilon), \log _{10}(B)$, and $\log _{10}\left(\mathrm{~d} \overline{\theta_{\rho}} / \mathrm{d} z\right)$ to find relationships of the form $\epsilon=10^{b} x^{m}$, where $m$ and $b$ are the slope and intercept of the line fit $\log _{10}(\epsilon)=m \log _{10}(x)+b$. (We perform these fits using logarithms in base 10 instead of natural logs simply for easier interpretation of the resulting plots.) a) $E$ vs Area

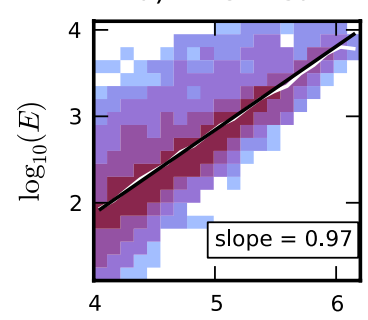

c) $D$ vs Area

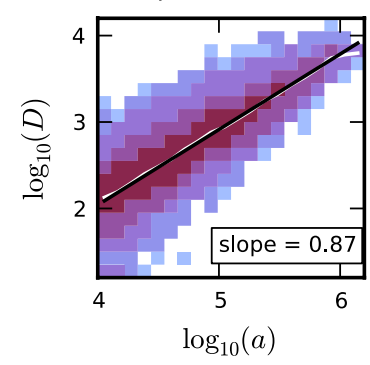

b) $E$ vs Perimeter

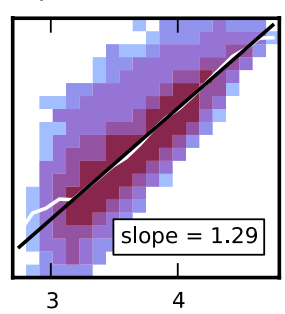

d) $D$ vs Perimeter

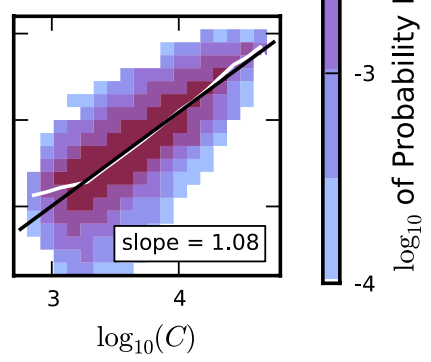

Fig. 10. Joint probability density function multiplied by bin area $(P(x, y) \Delta x \Delta y)$ for individual clouds in the combined BOMEX and ARM LES output of $\log _{10}(E)$ versus (a) $\log _{10}$ of crosssectional area, (b) $\log _{10}$ of cloud core perimeter, and $\log _{10}(D)$ versus (c) $\log _{10}$ of cross-sectional area, (d) $\log _{10}$ of cloud core perimeter. PDFs are plotted using a logarithmic scale. White lines indicate the mean $\log _{10}(E) / \log _{10}(D)$ value as a function of the $x$ axis variable, and black lines show linear least-square best fits of $\log _{10}(E) / \log _{10}(D)$ versus the $x$ axis variable.

The joint PDF of $\log _{10}(\epsilon)$ and $\log _{10}(B)$ shows a relationship with a great deal of variance (Fig. 11a). Nevertheless, the correlation between the variables of -0.78 is fairly high, and a linear fit with a slope of -1.29 does a reasonable job matching the data (RMS error of 0.46) except at small and large values of $B$ where $\epsilon$ is systematically underestimated.

The joint PDF of $\log _{10}(\epsilon)$ and $\log _{10}\left(\mathrm{~d} \overline{\theta_{\rho}} / \mathrm{d} z\right)$ shows a less robust linear relationship (correlation of -0.48 , Fig. 11b). There appear to be two regimes, one between $\log _{10}\left(\mathrm{~d} \overline{\theta_{\rho}} / \mathrm{d} z\right)$ values of -2.75 and -2 , and a second between -3 and -2.75 . The linear fit to this data (slope -1.35 , RMS error of 0.64 ) does reasonably well for larger stratification, but at low stratification $\epsilon$ is significantly underestimated.

Since the relationships between $\epsilon$ and $B$ and between $\epsilon$ and $\mathrm{d} \overline{\theta_{\rho}} / \mathrm{d} z$ have the same sense - stronger buoyancy and stratification mean weaker entrainment - we also try a fit to the product of the two variables. The joint PDF of $\log _{10}(\epsilon)$ and $\log _{10}\left(B \mathrm{~d} \overline{\theta_{\rho}} / \mathrm{d} z\right)$ shows a stronger linear relationship than either variable individually (correlation of -0.83 , Fig. 11c). The resulting curve fit (slope -1.06 , RMS error 0.35) still underestimates the entrainment rate at low and high values of buoyancy and stratification, but many of the extremely low entrainment values present at low buoyancy are raised by the addition of the stratification. Additionally, the fit is tantalizingly close to indicating a simple inverse relationship 

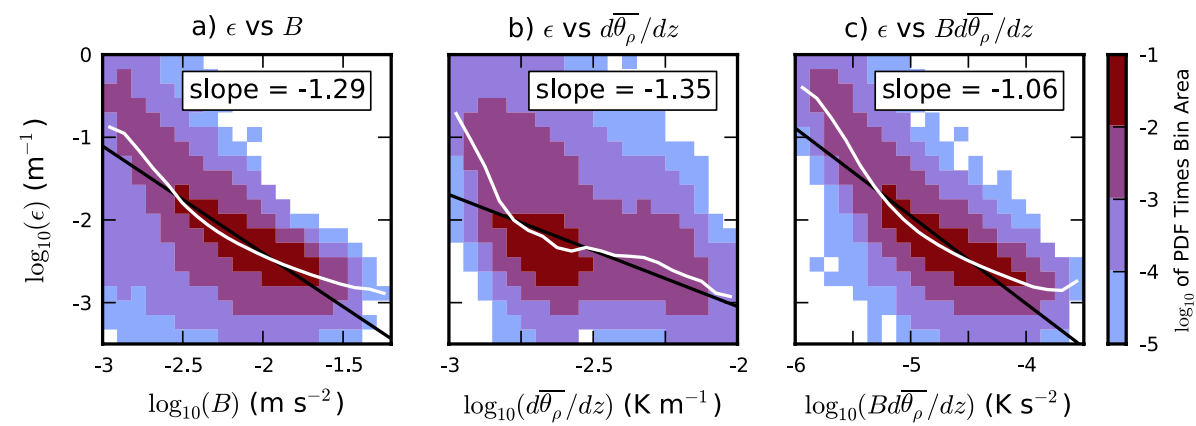

Fig. 11. Joint probability density functions multiplied by bin area $(P(x, y) \Delta x \Delta y)$ for individual clouds in the combined BOMEX and ARM LES output of $\log _{10}(\epsilon)$ versus (a) $\log _{10}$ of buoyancy, (b) $\log _{10}$ of environmental stratification, and (c) $\log _{10}$ of buoyancy times environmental stratification. PDFs are plotted using a logarithmic scale. White lines indicate the mean $\log _{10}(\epsilon)$ value as a function of the $x$ axis variable, and black lines show linear least-square best fits of $\log _{10}(\epsilon)$ versus the $x$ axis variable.

between $\epsilon$ and $B \mathrm{~d} \overline{\theta_{\rho}} / \mathrm{d} z$. We therefore conclude that a power law fit between $\epsilon$ and $B \mathrm{~d} \overline{\theta_{\rho}} / \mathrm{d} z$ provides a simple but skillful estimate of the entrainment rate of individual shallow cumulus clouds.

\subsection{Detrainment}

Again we repeat the previous analysis to examine the dependence of fractional detrainment $\delta$ on vertical velocity $w$ and critical mixing fraction $\chi_{\mathrm{c}}$. The joint PDF of $\log _{10}(\delta)$ and $\log _{10}(w)$ shows a relatively poor linear relationship (correlation -0.52 , Fig. 12a). However, the linear fit to this data shows a relatively good inverse relationship between the variables (slope -0.92, RMS error 0.34). However, unlike the other comparisons, the sense of the relationship between the variables has two regimes: $\delta$ decreases with increasing $w$ between $\log _{10}(w)$ values of -0.5 and 0.5 , but increases with $w$ above a $\log _{10}(w)$ of 0.5 .

The joint PDF of $\log _{10}(\delta)$ and $\log _{10}\left(\chi_{\mathrm{c}}\right)$ shows a much more linear relationship than $\log _{10}(w)$ (correlation -0.71 , Fig. 12b), but the curve fit between these variables is weaker (slope -1.34 , RMS error 0.62) and overestimates $\delta$ at small values of $\chi_{\mathrm{c}}$.

However, as with $\epsilon$, a fit between $\delta$ and the product $w \chi_{\mathrm{c}}$ does a better job than either alone. The joint PDF displays a stronger linear relationship than either variable alone (correlation -0.76 , Fig. 12c), and the curve fit matches the mean PDF values well (slope -0.86 , RMS error 0.32 ), also displaying a nearly inverse relationship between $\delta$ and $w \chi_{\mathrm{c}}$.

\subsection{Resulting best fits}

Figure 13 shows the resulting best-fit power-law curves for $\epsilon$ and $\delta$. We find the relationships

$\epsilon=5.19 \times 10^{-8}\left(B \mathrm{~d} \overline{\theta_{\rho}} / \mathrm{d} z\right)^{-1.07}$

and

$\delta=2.72 \times 10^{-3}\left(w \chi_{\mathrm{c}}\right)^{-0.89}$ provide reasonable fits to the individual cloud data. (The power law exponents for these fits are slightly different than those reported in the previous section, as we have expanded the data range over which we are performing these fits to include some of the more extreme model values.) As noted above, while suffering from a lack of dimensional consistency with $\epsilon$ and $\delta$, these parameterizations do have the intriguing property of nearly being a simple inverse relationship. In any case, these relationships provide a reasonable first-order estimate of the magnitude of $\epsilon$ and $\delta$ for individual cumulus clouds in the BOMEX and ARM cases.

However, large-scale models require parameterizations not of individual clouds, but of cloud ensembles. Translating these individual curve fits into equations usable for whole cloud fields is a problematic task. Formally, the ensemble entrainment and detrainment rates at a given height can be written in terms of $\epsilon\left(B, \mathrm{~d} \overline{\theta_{\rho}} / \mathrm{d} z\right)$ and $\delta\left(w, \chi_{\mathrm{c}}\right)$ as

$\epsilon_{\text {ensemble }}=$

$\frac{\int \rho w a \epsilon\left(B, \mathrm{~d} \overline{\theta_{\rho}} / \mathrm{d} z\right) P\left(w, a, B, \chi_{\mathrm{c}}, \mathrm{d} \overline{\theta_{\rho}} / \mathrm{d} z\right) \mathrm{d} w \mathrm{~d} a \mathrm{~d} B \mathrm{~d} \chi_{\mathrm{c}} \mathrm{d}\left(\mathrm{d} \overline{\theta_{\rho}} / \mathrm{d} z\right)}{\int \rho w a P\left(w, a, B, \chi_{\mathrm{c}}, \mathrm{d} \overline{\theta_{\rho}} / \mathrm{d} z\right) \mathrm{d} w \mathrm{~d} a \mathrm{~d} B \mathrm{~d} \chi_{\mathrm{c}} \mathrm{d}\left(\mathrm{d} \overline{\theta_{\rho}} / \mathrm{d} z\right)}$

and

$\delta_{\text {ensemble }}=$

$\frac{\int \rho w a \delta\left(w, \chi_{\mathrm{c}}\right) P\left(w, a, B, \chi_{\mathrm{c}}, \mathrm{d} \overline{\theta_{\rho}} / \mathrm{d} z\right) \mathrm{d} w \mathrm{~d} a \mathrm{~d} B \mathrm{~d} \chi_{\mathrm{c}} \mathrm{d}\left(\mathrm{d} \overline{\theta_{\rho}} / \mathrm{d} z\right)}{\int \rho w a P\left(w, a, B, \chi_{\mathrm{c}}, \mathrm{d} \overline{\theta_{\rho}} / \mathrm{d} z\right) \mathrm{d} w \mathrm{~d} a \mathrm{~d} B \mathrm{~d} \chi_{\mathrm{c}} \mathrm{d}\left(\mathrm{d} \overline{\theta_{\rho}} / \mathrm{d} z\right),}$

where $P\left(w, a, B, \chi_{\mathrm{c}}, \mathrm{d} \overline{\theta_{\rho}} / \mathrm{d} z\right)$ is the joint cloud core property PDF at the current height. The numerator of each equation is the sum of the mass entrainment $E$ or detrainment $D$ and the denominator is the total vertical mass flux $M=\rho a w$ of the cloud ensemble. Transformation of the individual cloud $\epsilon$ and $\delta$ into the ensemble values is thus a rather complex operation.

Instead of performing this complicated transformation, we simply refit $\epsilon\left(B, \mathrm{~d} \overline{\theta_{\rho}} / \mathrm{d} z\right)$ and $\delta\left(w, \chi_{\mathrm{c}}\right)$ using the ensemble values of $\epsilon, \delta$, and the mean cloud core properties. Figure 14 

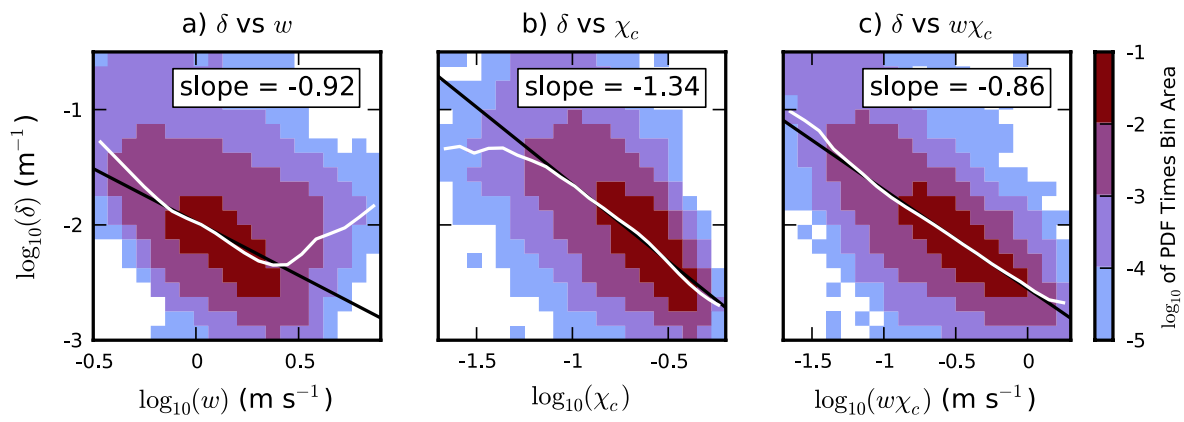

Fig. 12. Joint probability density functions multiplied by bin area $(P(x, y) \Delta x \Delta y)$ for individual clouds in the combined BOMEX and ARM LES output of $\log _{10}(\delta)$ versus (a) $\log _{10}$ of vertical velocity, (b) $\log _{10}$ of critical mixing fraction, and (c) $\log _{10}$ of vertical velocity times critical mixing fraction. PDFs are plotted using a logarithmic scale. White lines indicate the mean $\log _{10}(\delta)$ value as a function of the $x$ axis variable, and black lines show linear least-square best fits of $\log _{10}(\delta)$ versus the $x$ axis variable.

a) Entrainment

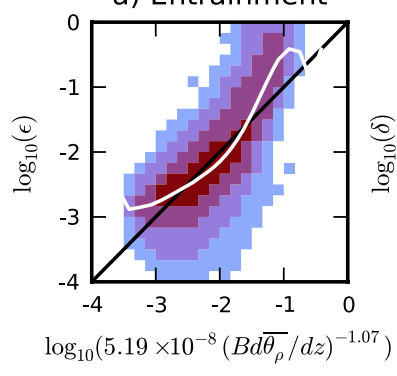

b) Detrainment

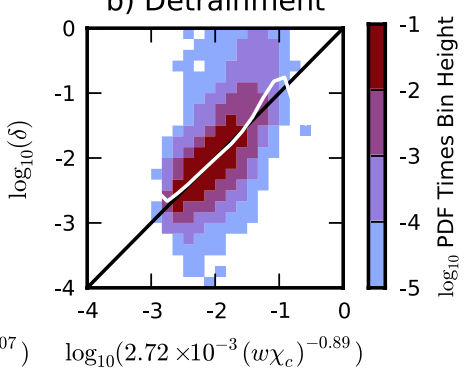

Fig. 13. Joint probability density functions multiplied by bin area $(P(x, y) \Delta x \Delta y)$ for individual clouds in the combined BOMEX and ARM LES output between fractional mass entrainment/detrainment rates and best-fit entrainment/detrainment rates as predicted by cloud core properties. (a) $\log _{10}(\epsilon)$ versus the best-fit $\epsilon\left(B, \mathrm{~d} \overline{\theta_{\rho}} / \mathrm{d} z\right)$ relationship. (b) $\log _{10}(\delta)$ versus the best-fit $\delta\left(w, \chi_{\mathrm{c}}\right)$ relationship. PDFs are plotted using a logarithmic scale. White lines indicate the mean $\log _{10}(\epsilon)$ or $\log _{10}(\delta)$ values as functions of the best-fit relationship, and black lines show the $\log _{10}(\epsilon)$ or $\log _{10}(\delta)$ values predicted by the best-fit relationship.

shows the resulting best-fit power-law curves for $\epsilon$ :

$\epsilon=4.2 \times 10^{-6}\left(B \mathrm{~d} \overline{\theta_{\rho}} / \mathrm{d} z\right)^{-0.70}$,

and for $\delta$ :

$\delta=3.76 \times 10^{-3}\left(w \chi_{\mathrm{c}}\right)^{-0.69}$.

Surprisingly, both fits display a $\approx-0.7$ power law, a coincidence for which we have no explanation.

\section{Discussion}

As far as we are aware, this study represents the first time PDFs of cloud core entrainment and detrainment with various other cloud core properties have been calculated for individual clouds in LES. This allows us to easily examine the a) Entrainment

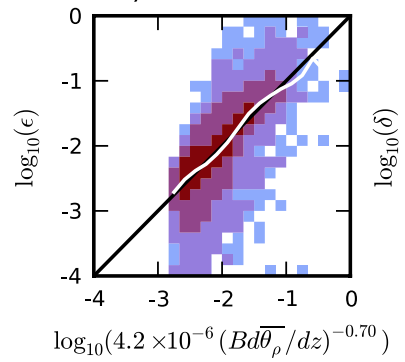

b) Detrainment

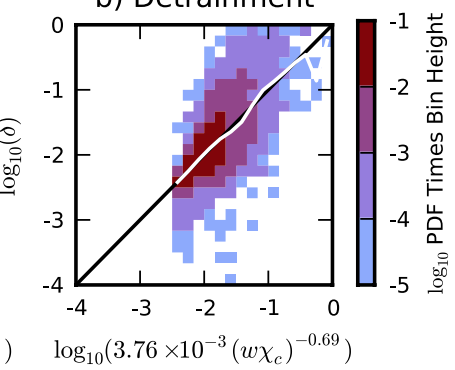

Fig. 14. Joint probability density functions multiplied by bin area $(P(x, y) \Delta x \Delta y)$ for the horizontal mean cloud ensembles of the BOMEX and ARM LES output between fractional mass entrainment/detrainment rates and best-fit entrainment/detrainment rates as predicted by cloud core properties. (a) $\log _{10}(\epsilon)$ versus the best-fit $\epsilon\left(B, \mathrm{~d} \overline{\theta_{\rho}} / \mathrm{d} z\right)$ relationship. (b) $\log _{10}(\delta)$ versus the best-fit $\delta\left(w, \chi_{\mathrm{c}}\right)$ relationship. PDFs are plotted using a logarithmic scale. White lines indicate the mean $\log _{10}(\epsilon)$ or $\log _{10}(\delta)$ values as functions of the best-fit relationship, and black lines show the $\log _{10}(\epsilon)$ or $\log _{10}(\delta)$ values predicted by the best-fit relationship.

behaviour of shallow cumulus ensembles and find novel results, such as the negligible role in vertical transport played by smaller cumulus, or the $C \propto a^{0.73}$ relationship between perimeter and area for clouds. Applying this technique to problems such as examining the cloud PDF changes that occur during the transition from shallow to deep convection would no doubt provide equally novel results.

Our analysis implies that $\delta$ is roughly inversely proportional to $w$, which is highly reminiscent of the multiparcel entrainment model of Neggers et al. (2002). While $w$ is not the strongest predictor of $\epsilon$ in our results, larger $w$ is undoubtedly associated with smaller $\epsilon$ (Fig. 5b), adding support to the Neggers et al. model. Romps and Kuang (2010) criticized the Neggers et al. model on the basis that cloud base properties are very uniform, so a dependence of entrainment rate on cloud properties would not produce the wide variance observed in cloud properties, proposing that stochastic 
entrainment events instead represent multiparcel entrainment better. Our results suggest that both models are partially correct, as $\epsilon$ and $\delta$ show strong dependence on cloud properties but also display a great deal of randomness (Fig. 13). For example, calculating the standard deviation of $\log _{10} \epsilon$ for moderate values of $B$ and $\mathrm{d} \overline{\theta_{\rho}} / \mathrm{d} z$ gives $\log _{10} \epsilon=(-2.0 \pm 0.3)$, equivalent to an $\epsilon$ range of $\approx(0.005-0.2) \mathrm{m}^{-1}$.

This creates the following picture of shallow cumulus dynamics: all clouds start with uniform properties at cloud base. Randomness in the mixing events experienced by each cloud produces property differences as the clouds rise, and these property differences then feed back upon the entrainment and detrainment rates. Entrainment events tend to decrease $B, w$ and $\chi_{\mathrm{c}}$, and reductions in $B, w$ and $\chi_{\mathrm{c}}$ all imply higher rates of entrainment and detrainment. A positive feedback thus appears to exist, so that clouds which experience large entrainment events early in their evolution tend to be more vulnerable to further dilution.

Our results have all been calculated for direct $\epsilon$ and $\delta$ values, which differ substantially from the $\epsilon$ and $\delta$ values needed for GCM parameterizations due to the influence of the moist cloud shell, Dawe and Austin (2011b). This makes it difficult to compare our results directly with previously published parameterizations, as we cannot be sure that differences between the parameterizations and our results are due to real differences or due to neglect of the modifying effects of the cloud shell. We hope to address this deficiency in a future paper.

However, some preliminary indications of the validity of cloud parameterizations can be drawn from our results. We find little dependence between $\epsilon$ and cloud area, contradicting parameterizations that vary entrainment rate with cloud radius (Arakawa and Schubert, 1974; Kain and Fritsch, 1990). This disagrees with the results of Stirling and Stratton (2012), who find a clear relationship with both $\epsilon$ and $\delta$ decreasing with mean area per cloud in simulations of the diurnal cycle of deep convection. However, Stirling and Stratton examine much larger clouds than our shallow cumulus, are looking at the behavior of the bulk cloud field rather than individual clouds, and measure entrainment with tracer budgets rather than direct calculations, which may help explain the discrepancy.

As assumed by buoyancy sorting parameterizations (Tiedtke, 1989; Bretherton and Park, 2009), critical mixing fraction $\chi_{\mathrm{c}}$ has strong effects on $\delta$, with large $\chi_{\mathrm{c}}$ suppressing detrainment. However, large $\chi_{\mathrm{c}}$ is also associated with reduced $\epsilon$, while buoyancy sorting parameterizations predict enhanced $\epsilon$. Other parameterizations relate $\epsilon$ to the quantity

$\epsilon=\frac{\alpha B}{w^{2}}-\frac{1}{w} \frac{\partial w}{\partial z}$

or some simplification thereof (de Rooy and Siebesma, 2010; Gregory, 2001). The inverse dependence of $\epsilon$ and $B$ do not rule out these types of relations, as $w$ and $B$ are correlated, and it is possible that a $B / w^{2}$ relationship could appear as a $1 / B$ due to these correlations. However, none of these relationships take into account the effect of background stratification, which appears to be important in our results and has also been incorporated into an entrainment parameterization by Stirling and Stratton (2012). Finally, as mentioned previously, we find support for the $w^{-1}$ parameterization of Neggers et al. (2002).

Finally, we acknowledge the awkwardness inherent in our parameterization relationships not being dimensionally consistent with the $\mathrm{m}^{-1}$ of $\epsilon$ and $\delta$. However, it is not clear that a complex turbulent phenomena like these should necessarily display dimensional consistency; the $C \propto a^{0.73}$ relationship we find for the dependence of cross-sectional area and perimeter certainly does not, for example. Either way, we believe the main value of these results is as a guide to developing better parameterizations, acting as a statistical null hypothesis that a mass entrainment and detrainment scheme should better in terms of larger correlations and smaller RMS error.

\section{Conclusions}

Joint probability density functions for fractional cloud core mass entrainment/detrainment rates and horizontal mean cloud core properties were calculated for individual clouds isolated from BOMEX and ARM LES with a cloud tracking algorithm. Clouds with cross-sectional cloud core area $a$ less than $10000 \mathrm{~m}^{2}$ were found to have negligible effects on the vertical mass and property transports of the cloud field despite occupying $\approx 50 \%$ of the total cloud core area, and were excluded from the analysis.

PDFs of cloud core properties showed most properties having normal distributions, with the exception of cloud core area, which was exponentially distributed, and fractional entrainment and detrainment rates, which displayed log-normal distributions. Joint PDFs between $\epsilon$ and $\delta$ with $a$ showed little dependency of fractional entrainment or detrainment rates on cloud core area. Examination of the relationship of cloud core cross-sectional perimeter and area showed $C \propto a^{0.73}$.

Dependence between $\epsilon, \delta$ and various cloud core properties was quantified using mutual information. $\epsilon$ was found to have the highest MI with $B$ and $\mathrm{d} \overline{\theta_{\rho}} / \mathrm{d} z$, and $\delta$ with $\chi_{\mathrm{c}}$ and $w$. Overall, $\epsilon$ and $\delta$ appear to be primarily governed by buoyancy, either directly or through the critical mixing fraction. Highly buoyant clouds experience less fractional entrainment and detrainment than less buoyant clouds. Similarly, highly stratified environments reduce cloud core entrainment and large upward velocities reduce cloud core detrainment. Power law fits of the form $\epsilon\left(B, \mathrm{~d} \overline{\theta_{\rho}} / \mathrm{d} z\right)$ and $\delta\left(w, \chi_{c}\right)$ were found to provide reasonable predictions of entrainment/detrainment rates both for individual clouds and the overall cloud ensemble. However, this study has only examined two shallow cumulus regimes; a bigger parameter space is needed to validate these results. 
We have not directly compared the results presented here with entrainment and detrainment rate parameterizations in the literature, primarily because these parameterizations have been tuned to values derived from tracer budget calculations, which are more applicable to ensemble cloud rate calculations needed for GCMs. Performing this calculation requires correcting the direct entrainment/detrainment rates for the effect the moist cloud shell has on tracer fluxes. Evaluating these equivalent tracer budget rates and comparing them to cloud parameterizations currently in use would help understand how applicable these results are to GCM parameterizations.

\section{Appendix A}

\section{Mutual information}

Shannon and Weaver (1949) defined the information content of a single measurement of a variable $x$ with probability density function $P(x)$ to be

$-\ln (P(x))$,

and the entropy $H$ of the variable's PDF to be

$H=-\int P(x) \ln (P(x)) \mathrm{d} x$.

The entropy is a measure of how much information is required to represent the PDF.

The mutual information $I(X ; Y)$, where $X$ and $Y$ are any two random variables, is the entropy of $X$ minus the entropy of $X$ conditioned on $Y$ :

$I(X ; Y)=H(X)-H(X \mid Y)$.

Mutual information may be thought of as the information in $\mathrm{X}$ that is shared with $Y$ - the information in $X$ that remains once the information that $X$ does not share with $Y$ is removed. Combining Eqs. (A2) and (A3) gives

$I(X ; Y)=-\int[P(x) \ln (P(x))-P(x, y) \ln (P(x \mid y))] \mathrm{d} x \mathrm{~d} y$.

Since $P(x \mid y)=P(x, y) / P(y)$, this can be simplified to

$I(X ; Y)=\int P(x, y) \ln \left(\frac{P(x, y)}{P(x) P(y)}\right) \mathrm{d} x \mathrm{~d} y$.

Mutual information is symmetric $(I(X ; Y)=I(Y ; X))$, and can range in value from zero when $X$ and $Y$ are completely independent $(P(x, y)=P(x) P(y))$, to $H(X)$ when $X$ and $Y$ are perfectly correlated $(P(x, y)=P(x)=P(y))$.

The conditional mutual information $I(X ; Y \mid Z)$ is an extension of mutual information that measures the information that remains shared between variables $X$ and $Y$ once the information they share with a third variable $Z$ has been removed:

$I(X ; Y \mid Z)=H(X \mid Z)-H(X \mid(Y, Z))$.
Combining Eqs. (A2) and (A6) gives

$$
\begin{aligned}
& I(X ; Y \mid Z)=-\int[P(x, z) \ln (P(x \mid z)) \\
& \quad-P(x, y, z) \ln (P(x \mid(y, z))] \mathrm{d} x \mathrm{~d} y \mathrm{~d} z .
\end{aligned}
$$

Since $P(x \mid(y, z))=P(x, y, z) / P(y, z) \quad$ and $\quad P(x \mid z)=$ $P(x, z) / P(z)$, this can be simplified to

$I(X ; Y \mid Z)=\int P(x, y, z) \ln \left(\frac{P(z) P(x, y, z)}{P(x, z) P(y, z)}\right) \mathrm{d} x \mathrm{~d} y \mathrm{~d} z$.

Acknowledgements. Support for this research was provided by the Canadian Foundation for Climate and Atmospheric Science through the Cloud Aerosol Feedback and Climate network. We thank Marat Khairoutdinov for making SAM available to the cloud modelling community. Figures were generated using the matplotlib library (Hunter, 2007) in the Python programming language.

Edited by: G. Feingold

\section{References}

Arakawa, A. and Schubert, W. H.: Interaction of a cumulus cloud ensemble with the large-scale environment, part I, J. Atmos. Sci., 31, 674-701, 1974.

Bechtold, P., Köhler, M., Jung, T., Doblas-Reyes, F., Leutbecher, M., Rodwell, M. J., Vitart, F., and Balsamo, G.: Advances in simulating atmospheric variability with the ECMWF model: from synoptic to decadal time-scales, Q. J. Roy. Meteor. Soc., 134, 1337-1351, doi:10.1002/qj.289, 2008.

Bretherton, C. S. and Park, S.: A new bulk shallow-cumulus model and implications for penetrative entrainment feedback on updraft buoyancy, J. Atmos. Sci., 65, 2174-2193, doi:10.1175/2007JAS2242.1, 2008.

Bretherton, C. S. and Park, S.: A new moist turbulence parameterization in the community atmosphere model, J. Climate, 22, 3422-3448, doi:10.1175/2008JCLI2556.1, 2009.

Bretherton, C. S., McCaa, J. R., and Grenier, H.: A new parameterization for shallow cumulus convection and its application to marine subtropical cloud-topped boundary layers. Part I: description and 1-D results., Mon. Weather Rev., 132, 864-882, doi:10.1175/1520-0493(2004)132<0883:ANPFSC > 2.0.CO;2, 2004.

Brown, A. R., Cederwall, R. T., Chlond, A., Duynkerke, P. G., Golaz, J.-C., Khairoutdinov, M. F., Lewellen, D. C., Lock, A. P., Macvean, M. K., Moeng, C.-H., Neggers, R. A. J., Siebesma, A. P., and Stevens, B.: Large-eddy simulation of the diurnal cycle of shallow cumulus convection over land, Q. J. Roy. Meteor. Soc., 128, 1075-1093, doi:10.1256/003590002320373210, 2002.

Dawe, J. T. and Austin, P. H.: Interpolation of LES cloud surfaces for use in direct calculations of entrainment and detrainment, Mon. Weather Rev., 139, 444-456, 2011a.

Dawe, J. T. and Austin, P. H.: The influence of the cloud shell on tracer budget measurements of LES cloud entrainment, J. Atmos. Sci., 68, 2909-2920, doi:10.1175/2011JAS3658.1, 2011b. 
Dawe, J. T. and Austin, P. H.: Statistical analysis of an LES shallow cumulus cloud ensemble using a cloud tracking algorithm, Atmos. Chem. Phys., 12, 1101-1119, doi:10.5194/acp-12-11012012, 2012.

de Rooy, W. C. and Siebesma, A. P.: A simple parameterization for detrainment in shallow cumulus, Mon. Weather Rev., 136, 560576, 2008.

de Rooy, W. C. and Siebesma, A. P.: Analytical expressions for entrainment and detrainment in cumulus convection, Q. J. Roy. Meteor. Soc., 136, 1216-1227, doi:10.1002/qj.640, 2010.

de Rooy, W. C., Bechtold, P., Fröhlich, K., Hohenegger, C., Jonker, H., Mironov, B., Siebesma, A. P., Teixeira, J., and Yano, J.-I.: Entrainment and detrainment in cumulus convection: an overview, Q. J. Roy. Meteor. Soc., 139, 1-19, doi:10.1002/qj.1959, 2013.

Gregory, D.: Estimation of entrainment rate in simple models of convective clouds, Q. J. Roy. Meteor. Soc., 127, 53-72, 2001.

Hunter, J. D.: Matplotlib: a 2D graphics environment, Comput. Sci. Eng., 9, 90-95, doi:10.1109/MCSE.2007.55, 2007.

Kain, J. S. and Fritsch, J. M.: A one-dimensional entraining/detraining plume model and its application in convective parameterization, J. Atmos. Sci., 47, 2784-2802, 1990.

Khairoutdinov, M. F. and Randall, D. A.: Cloud resolving modeling of the ARM summer 1997 IOP: model formulation, results, uncertainties, and sensitivities, J. Atmos. Sci., 60, 607-625, 2003.

Klocke, D., Pincus, R., and Quaas, J.: On constraining estimates of climate sensitivity with present-day observations through model weighting, J. Climate, 24, 6092-6099, doi:10.1175/2011JCLI4193.1, 2011.

Neggers, R. A. J., Siebesma, A. P., and Jonker, H. J. J.: A multiparcel model for shallow cumulus convection, J. Atmos. Sci., 59, 1655-1668, 2002.

Randall, D., Krueger, S., Bretherton, C., Curry, J., Duynkerke, P., Moncrieff, M., Ryan, B., Starr, D., Miller, M., Rossow, W., Tselioudis, G., and Wielicki, B.: Confronting models with data: the GEWEX cloud systems study, B. Am. Meteorol. Soc., 84, 455469, doi:10.1175/BAMS-84-4-455, 2003.

Romps, D. M.: A direct measure of entrainment, J. Atmos. Sci., 67, 1908-1927, 2010.

Romps, D. M. and Kuang, Z.: Nature versus nurture in shallow convection, J. Atmos. Sci., 67, 1655-1666, doi:10.1175/2009JAS3307.1, 2010.

Sanderson, B. M., Knutti, R., Aina, T., Christensen, C., Faull, N., Frame, D. J., Ingram, W. J., Piani, C., Stainforth, D. A., Stone, D. A., and Allen, M. R.: Constraints on model response to greenhouse gas forcing and the rold of subgrid-scale processes, J. Climate, 21, 2384-2400, doi:10.1175/2008JCLI1869.1, 2008.

Shannon, C. E. and Weaver, W.: The Mathematical Theory of Communication, University of Illinois Press, 1949.
Siebesma, A. P.: Shallow cumulus convection, in: Buoyant Convection in Geophysical Flows, edited by: Plate, E. J., Kluwer Academic Publishers, 441-486, 1998.

Siebesma, A. P. and Cuijpers, J. W. M.: Evaluation of parametric assumptions for shallow cumulus convection, J. Atmos. Sci., 52, 650-666, 1995.

Siebesma, A. P. and Jonker, H. J. J.: Anomalous Scaling of Cumulus Cloud Boundaries, Phys. Rev. Lett., 85, 214-217, 2000.

Siebesma, A. P., Bretherton, C. S., Brown, A., Chlond, A., Cuxart, J., Duynkerke, P. G., Jiang, H., Khairoutdinov, M., Lewellen, D., Moeng, C.-H., Sanchez, E., Stevens, B., and Stevens, D. E.: A large eddy simulation intercomparison study of shallow cumulus convection, J. Atmos. Sci., 60, 1201-1219, doi:10.1175/1520-0469(2003)60<1201:ALESIS > 2.0.CO;2, 2003.

Stevens, B.: Atmospheric moist convection, Annu. Rev. Earth Pl. Sc., 33, 605-643, doi:10.1146/annurev.earth.33.092203.122658, 2005.

Stevens, B., Ackerman, A. S., Albrecht, B. A., Brown, A. R., Chlond, A., Cuxart, J., Duynkerke, P. G., Lewellen, D. C., Macvean, M. K., Neggers, R. A. J., Sanchez, E., Siebesma, A. P., and Stevens, D. E.: Simulations of trade wind cumuli under a strong inversion, J. Atmos. Sci., 58, 1870-1891, 2001.

Stirling, A. J. and Stratton, R. A.: Entrainment processes in the diurnal cycle of deep convection over land, Q. J. Roy. Meteor. Soc., 138, 1135-1149, doi:10.1002/qj.1868, 2012.

Tiedtke, M.: A comprehensive mass flux scheme for cumulus parameterization in large-scale models, Mon. Weather Rev., 117, 1779-1800, 1989.

Turner, J. S.: The motion of buoyant elements in turbulent surroundings, J. Fluid Mech., 16, 1-16, 1963.

van Zanten, M. C., Stevens, B., Nuijens, L., Siebesma, A. P., Ackerman, A. S., Burnet, F., Cheng, A., Couvreux, F., Jiang, H., Khairoutdinov, M., Kogan, Y., Lewellen, D. C., Mechem, D., Nakamura, K., Noda, A., Shipway, B. J., Slawinska, J., Wang, S., and Wyszogrodzki, A.: Controls on precipitation and cloudiness in simulations of trade-wind cumulus as observed during RICO, J. Adv. Model. Earth Syst., 3, M06001, doi:10.1029/2011MS000056, 2011.

von Salzen, K. and McFarlane, N. A.: Parameterization of the bulk effects of lateral and cloud-top entrainment in transient shallow cumulus clouds, J. Atmos. Sci., 59, 1405-1430, doi:10.1175/1520-0469(2002)059<1405:POTBEO > 2.0.CO;2, 2002.

Wagner, T. M. and Graf, H.-F.: An ensemble cumulus convection parameterization with explicit cloud treatment, J. Atmos. Sci., 67, 3854-3869, doi:10.1175/2010JAS3485.1, 2010. 